\title{
Stabilization of the viscoelastic Euler-Bernoulli type equation with a local nonlinear dissipation
}

\author{
Tae Gab Ha and Jong Yeoul Park
}

Communicated by Y. Charles Li, received October 23, 2009.

\begin{abstract}
In this paper, we consider the viscoelastic Euler-Bernoulli type equation

$$
u_{t t}+\Delta^{2} u-M\left(\|\nabla u\|^{2}\right) \Delta u-\int_{0}^{t} g(t-\tau) \Delta^{2} u(\tau) d \tau+\rho\left(x, u_{t}\right)=0 .
$$

This work is devoted to prove the existence of global solutions and decay for the energy of solutions of the Euler-Bernoulli type equation with nonlinear localized dissipation term.
\end{abstract}

\section{Contents}

1. Introduction 335

2. Notations and main results 337

3. Existence of solutions 340

4. Energy decay 346

References $\quad 365$

\section{Introduction}

In this paper, we are concerned with global existence and decay for the energy of solutions of viscoelastic Euler-Bernoulli type equation with a localized damping term:

$$
\begin{aligned}
& \left\{\begin{array}{l}
u_{t t}+\Delta^{2} u-M\left(\|\nabla u\|^{2}\right) \Delta u-\int_{0}^{t} g(t-\tau) \Delta^{2} u(\tau) d \tau+\rho\left(x, u_{t}\right)=0 \quad \text { in } \quad \Omega \times \mathbb{R}_{+}, \\
u=\frac{\partial u}{\partial \nu}=0 \quad \text { on } \quad \Gamma \times \mathbb{R}_{+}, \\
u(x, 0)=u_{0}(x), \quad u^{\prime}(x, 0)=u_{1}(x), \quad x \in \Omega
\end{array}\right.
\end{aligned}
$$

2000 Mathematics Subject Classification. 35B40, 35L75, 74Dxx.

Key words and phrases. Existence of solution, energy decay, Euler-Bernoulli equation, local dissipation. 
where $\Omega \subset \mathbb{R}^{n}$ is an bounded domain, $n \geq 1$, with boundary $\Gamma=\Gamma_{0} \cup \Gamma_{1}$ of class $C^{2}$, where $\Gamma_{0}$ and $\Gamma_{1}$ are closed and disjoint and $M \in C^{1}\left(\mathbb{R}_{+}\right) . g(s)$ is a bounded $C^{2}$ function and $\rho(x, s)$ is almost everywhere differentiable and nondecreasing function in $s$. We shall denote by $\nu$ the unit outward normal vector to $\Gamma$. $\Delta$ and $\nabla$ stand for the Laplacian and gradian with respect to the spatial variables respectively, ' denotes the derivative with respect to time $t$, and $\mathbb{R}_{+}=[0, \infty)$.

The problem of proving existence of solutions has been studied from old times. There are many methods to solve existence of solutions, but recently many authors use the Galerkin's method. This paper is used Galerkin's method solving existence of solutions, too.

The problem of stabilization of partial differential equation has recently attracted a lot of attention and various results are available (see [1], [2], [4], [8], $[\mathbf{9}],[\mathbf{1 0}],[\mathbf{1 8}],[\mathbf{1 9}],[\mathbf{2 0}],[\mathbf{2 1}])$. When $\rho \equiv 0$, the problem has been treated many authors (cf. [8], [9], [10], [20] and a list of references therein). However, this paper put great emphasis on $\rho\left(x, u_{t}\right)$ term.

For the case of wave equation, Zuazua [22] had treat the linear case $\rho(x, v)=$ $a(x) v$ with $a(x)$ vanishing somewhere on $\bar{\Omega}$. Zuazua proved that any energy finite solution $u(t)$ satisfies the exponential decay

$$
E(t) \leq C E(0) e^{-\lambda t}
$$

for some $\lambda>0$. For the nonlinear case of $\rho(x, v)$ like $\rho(x, v)=a(x)|v|^{r} v$, Nakao has many treated (cf. [14], [15], [16]). In this case, the energy of solutions goes to zero, as $t \rightarrow \infty$, with a polynomial rate of decay.

For the case of Euler-Bernoulli type equation, Tucsnak [21] studied the linear case $\rho(x, v)=a(x) v$. By using appropriate Lyapounov functional, Tucsnak [21] found the result like Zuazua [22]. For the nonlinear case, Cavalcanti et al. [3] considered the following problem

$$
u_{t t}+\Delta^{2} u-\int_{0}^{t} g(t-\tau) \Delta^{2} u(\tau) d \tau+a(t) u_{t}=0 \quad \text { in } \quad \Omega \times(0, \infty),
$$

where $a(t)$ is a nonlocal nonlinearity type function. In this case, $M \equiv 0$ in (1.1). Using the perturbed energy method by constructing a suitable Liapunov functional, [3] proved the exponential energy of the Euler-Bernoulli equation with a nonlocal dissipation in general domains. And Charão et al. [4] considered

$$
\left\{\begin{array}{l}
u_{t t}+\Delta^{2} u-\alpha\left(\int_{\Omega}|\nabla u|^{2} d x\right) \Delta u+\rho\left(x, u_{t}\right)=0 \quad \text { in } \quad \Omega \times(0, \infty), \\
u=\frac{\partial u}{\partial \eta}=0, \quad \text { on } \quad \Gamma \times(0, \infty),
\end{array}\right.
$$

where $\alpha$ is a positive constant and $\rho\left(x, u_{t}\right)$ is a localized damping term. In this case, $M$ is a constant and $g \equiv 0$ in (1.1). By using the Nakao's lemma, it was proved that polynomial decay rate of solution.

This paper leads to special difference inequalities for the energy of solutions and allows to apply the method developed in [4] and [14]. However, method of $[\mathbf{1 4}]$ produces some lower order terms that we manage with compactness. In order to obtain some identities, [4] and [14] were used the multiplier technique but the multiplier method is not suitable when dealing with the memory term $\int_{0}^{t} g(t-$ $\tau) \Delta^{2} u(\tau) d \tau$. To overcome this point we use well-known inequalities and Sobolev imbedding theorem properly. The problem is then reduced to showing that the unique solution of (1.1) such that $u \equiv 0$ in $\omega \times \mathbb{R}_{+}$is the trivial one, which requires 
the application of a unique continuation result in [7]. At this point, we observe that the unique continuation result in [7] applies only when $\omega$ is neighborhood of the whole boundary, which leads us to require such assumption in our present proofs. In other words, the decay of solutions of (1.1) is obtained localizing the damping function in a neighborhood of the whole boundary.

To prove the decay rats of the energy

$$
E(t)=\frac{1}{2} \int_{\Omega}\left|u^{\prime}\right|^{2} d x+\frac{1}{2} \int_{\Omega}|\Delta u|^{2} d x+\frac{1}{2} \hat{M}\left(\|\nabla u\|^{2}\right),
$$

where

$$
\hat{M}(t)=\int_{0}^{t} M(s) d s
$$

we need to define a modified energy function. Indeed, a formal computation gives

$$
E^{\prime}(t)=-\left(\rho\left(x, u^{\prime}\right), u^{\prime}\right)+\int_{0}^{t} g(t-\tau)\left(\Delta u(\tau), \Delta u^{\prime}(t)\right) d \tau,
$$

which shows that we do not have any information about the sign of $E^{\prime}(t)$. To solve this problem we use an argument from Dafermos [5] to define a new energy function $e(t)$ such that $e^{\prime}(t) \leq 0$ and $E(t) \leq C e(t)$ for some positive constant $C$. This will be discussed in section 4 .

This paper is organized as follows: In section 2, we recall the notation and hypotheses and introduce our main results and lemma to prove our main results. In section 3, using the Galerkin's method we prove the existence and uniqueness of regular and weak solutions to problem (1.1). In section 4, we estimates some identities and inequalities and then using lemmas, we prove the energy decay.

\section{Notations and main results}

We begin this section introducing some notations and our main results. Throughout this paper we define $V=\left\{v \in H^{2}(\Omega) ; v=\frac{\partial v}{\partial \nu}=0\right.$ on $\left.\Gamma\right\}$ equipped with the norm $\|v\|_{V}=\|\Delta v\|$, where $\|\cdot\|$ is a $L^{2}$-norm, $W=\left\{v \in V ; \Delta^{2} v \in L^{2}(\Omega)\right\}$ equipped with the norm $\|w\|_{W}=\|w\|_{V}+\left\|\Delta^{2} w\right\|$ and $(u, v)=\int_{\Omega} u(x) v(x) d x$. From the Poincare's inequality, it follows that $\|\cdot\|_{V}$ and $\|\cdot\|_{W}$ are equivalent to the standard norms of $H^{2}(\Omega)$ and $H^{4}(\Omega)$, respectively. Now we give the hypotheses for the main results.

$\left(\mathbf{H}_{1}\right)$ Hypotheses on $\Omega$.

Let $\Omega \subset \mathbb{R}^{n}$ be an bounded domain, $n \geq 1$, with boundary $\Gamma=\Gamma_{0} \cup \Gamma_{1}$ of class $C^{2}$. Here $\Gamma_{0}$ and $\Gamma_{1}$ are closed and disjoint, $\Gamma_{0} \neq \emptyset$, satisfying the following condition:

$$
\begin{aligned}
& m \cdot \nu \geq \delta>0 \text { on } \Gamma_{1}, \quad m \cdot \nu \leq 0 \text { on } \Gamma_{0}, \\
& m(x)=x-x^{0}\left(x^{0} \in \mathbb{R}^{n}\right) \text { and } R=\max _{x \in \bar{\Omega}}|m(x)|,
\end{aligned}
$$

where $\nu$ represents the unit outward normal vector to $\Gamma$.

$\left(\mathbf{H}_{2}\right)$ Hypotheses on $\mathbf{M}$.

We consider $M$ is a real-valued nondecreasing function satisfying the conditions

$$
M \in C^{1}\left(\mathbb{R}_{+}\right) \quad \text { and } \quad M(s) \geq s_{0}>0 \quad \text { for all } \quad s \geq 0 .
$$

$\left(\mathbf{H}_{3}\right)$ Hypotheses on $\mathbf{g}$.

We assume the $g: \mathbb{R}_{+} \rightarrow \mathbb{R}_{+}$is a bounded $C^{2}$ function satisfying

$$
1-\int_{0}^{\infty} g(s) d s=\ell>0
$$


and such that

$$
\begin{gathered}
-c_{1} g(t) \leq g^{\prime}(t) \leq-c_{2} g(t), \\
0 \leq g^{\prime \prime}(t) \leq c_{3} g(t)
\end{gathered}
$$

where $c_{1}, c_{2}$ and $c_{3}$ are positive constants.

$\left(\mathbf{H}_{4}\right)$ Hypotheses on $\rho$.

Let $\rho(x, s)$ is almost everywhere differentiable and nondecreasing function in $s$ and satisfies

$$
\begin{array}{ll}
c_{4} a(x)|s|^{r+1} \leq|\rho(x, s)| \leq c_{5} a(x)\left(|s|^{r+1}+|s|\right), & \text { if } \quad|s| \leq 1 \\
\text { and } & \\
c_{6} a(x)|s|^{p+1} \leq|\rho(x, s)| \leq c_{7} a(x)\left(|s|^{p+1}+|s|\right), & \text { if } \quad|s| \geq 1,
\end{array}
$$

where $c_{4}, c_{5}, c_{6}$ and $c_{7}$ are positive constants, $-1<r<\infty,-1<p \leq \frac{2}{n-2}$ if $n \geq 3$ $(-1<p<\infty$ if $n=1,2)$. Also $a(x) \in L^{\infty}(\Omega)$ satisfies

$$
a(x) \geq a_{0}>0 \quad \text { on } \quad \omega,
$$

where $\omega$ is a neighborhood of $\Gamma$.

In addition, we assume that

$$
\begin{aligned}
& \rho(x, s) s \geq 0 \quad \text { and } \quad \frac{\partial \rho(x, s)}{\partial s} \geq 0, \quad \text { for all } \quad(x, s) \in \Omega \times \mathbb{R} ; \\
& \rho(\cdot, s) \quad \text { and } \quad \frac{\partial \rho(\cdot, s)}{\partial s} \in C(\bar{\Omega}) .
\end{aligned}
$$

A typical example of $\rho(x, s)$ is

$$
\rho(x, s)=\left\{\begin{array}{lll}
a(x) L^{-r-1}|s|^{r} s & \text { if } & |s| \leq L \\
a(x) L^{-p-1}|s|^{p} s & \text { if } & |s| \geq L
\end{array}\right.
$$

with $L>0$ (cf. [14]).

Now, we are in a position to state our main results.

Theorem 2.1. Let the initial data $\left\{u_{0}(x), u_{1}(x)\right\}$ belong to $V \times L^{2}(\Omega)$ and assume that $\left(H_{1}\right)-\left(H_{4}\right)$ hold. Then problem (1.1) admits an unique weak solution $u$ having the regularity

$$
u \in C\left(\mathbb{R}_{+} ; V\right) \cap C^{1}\left(\mathbb{R}_{+} ; L^{2}(\Omega)\right) .
$$

If we show Theorem 2.1, then we can assume the following hypothesis.

$\left(\mathbf{H}_{5}\right)$

Let $u$ is a solution of (1.1) and for any $\Phi \in W^{1, \infty}\left(\mathbb{R}_{+}\right), \Psi \in L^{\infty}\left(\mathbb{R}_{+}\right), \alpha \in \mathbb{R}_{+}$, the only function $v \in L^{2}\left(\Omega \times \mathbb{R}_{+}\right)$satisfies the conditions

$$
\left\{\begin{array}{l}
v_{t t}+\Delta^{2} v-\Phi(t) \Delta v-\alpha \Delta^{2} u-\int_{0}^{t} \Psi(t-\tau) \Delta^{2} u(\tau) d \tau=0 \quad \text { in } \quad \Omega \times \mathbb{R}_{+}, \\
v=\frac{\partial v}{\partial \nu}=0 \quad \text { on } \Gamma \times \mathbb{R}_{+}, \\
v=0 \quad \text { in } \quad \omega \times \mathbb{R}_{+},
\end{array}\right.
$$

then $u=v \equiv 0$ in $\Omega \times \mathbb{R}_{+}$.

Remark 2.1. For $\Phi \in W^{1, \infty}\left(\mathbb{R}_{+}\right)$and $\Psi \in L^{\infty}\left(\mathbb{R}_{+}\right),\left(H_{5}\right)$ holds true at least if $\Gamma_{1}=\Gamma$ (which can be true for star-shaped domains), according to [7], [21]. Moreover, if $\Omega$ is an interval of the real line $\left(H_{5}\right)$ holds for any open subset $\omega \subset \Omega$ (cf. [6]). 
In order to state another main result, we define the associated energy of problem (1.1) by

where

$$
E(t)=\frac{1}{2} \int_{\Omega}\left|u^{\prime}\right|^{2} d x+\frac{1}{2} \int_{\Omega}|\Delta u|^{2} d x+\frac{1}{2} \hat{M}\left(\|\nabla u\|^{2}\right),
$$

$$
\hat{M}(t)=\int_{0}^{t} M(s) d s .
$$

Theorem 2.2. Let $\left(u_{0}(x), u_{1}(x)\right) \in V \times L^{2}(\Omega)$ and $R>0$ such that

$$
\left\|\left(u_{0}(x), u_{1}(x)\right)\right\|_{V \times L^{2}(\Omega)} \leq R
$$

. Then the energy $E(t)$ associated with the solutions of (1.1) has the decay property

$$
E(t) \leq C(1+t)^{-\eta_{1}}, \quad(i=1,2,3,4),
$$

where $C=C(R, E(0))$ is a positive constant and the decay rate $\eta$ is given as follows corresponding to the cases;

case 1

If $r \geq 0$ and $0 \leq p \leq \frac{2}{n-2}$, then $\eta_{1}=\frac{2}{r}$.

case 2

If $r \geq 0$ and $-1<p<0$, then

$$
\eta_{2}=\min \left\{\frac{2}{r+2}, \frac{1}{p+2}\right\} \text {. }
$$

case 3

If $-1<r<0$ and $0 \leq p \leq \frac{2}{n-2}$, then

case 4

$$
\eta_{3}=\min \left\{\frac{1}{r+1}, \frac{-r}{2(r+1)}\right\} \text {. }
$$

If $-1<r<0$ and $-1<p<0$, then

$$
\eta_{4}=\min \left\{\frac{1}{r+1}, \frac{-r}{2(r+1)}, \frac{1}{p+1}\right\} .
$$

In order to prove of above theorem, we need the following lemmas.

Lemma 2.1. (Gagliardo-Nirenberg). Let $1 \leq r<p<\infty, 1 \leq q \leq p$ and $0 \leq m$. Then,

$$
\|v\|_{W^{k, p}} \leq C\|v\|_{W^{m, q}}^{\theta}\|v\|_{L^{r}}^{1-\theta}
$$

for $v \in W^{m, p}(\Omega) \cap L^{r}(\Omega), \Omega \subset \mathbb{R}^{N}$, where $C$ is a positive constant and

$$
\theta=\left(\frac{k}{N}+\frac{1}{r}-\frac{1}{p}\right)\left(\frac{m}{N}+\frac{1}{r}-\frac{1}{q}\right)^{-1}
$$

provided that $0<\theta \leq 1$.

Lemma 2.2. (Nakao $[\mathbf{1 3}]$ ) Let $\phi(t)$ be a nonnegative function on $\mathbb{R}_{+}$satisfying

$$
\sup _{t \leq s \leq t+T} \phi(s)^{1+\gamma} \leq \psi(t)\{\phi(t)-\phi(t+T)\}
$$

with $T>0, \gamma>0$ and $\psi(t)$ a nondecreasing continuous function. Then $\phi(t)$ has the decay property

$$
\phi(t) \leq\left\{\phi(0)^{-\gamma}+\int_{T}^{t} \psi(s)^{-1} d s\right\}^{\frac{-1}{\gamma}} \quad \text { for } \quad t \geq T
$$


If $\gamma=0$ in the above we have

$$
\phi(t) \leq C \phi(0) e^{-\lambda t}
$$

for some $\lambda>0$.

\section{Existence of solutions}

In this section we prove the existence and uniqueness of regular and weak solutions to problem (1.1). Firstly we consider regular solutions and then, using density arguments we extend the same results for weak solutions.

Let us solve the variational problem associated with (1.1), which is given by: find $u(t) \in W$ such that

$$
\begin{aligned}
\left(u_{t t}(t), w\right)+(\Delta u(t), \Delta w)+ & M\left(\|\nabla u\|^{2}\right)(\nabla u(t), \nabla w) \\
& -\int_{0}^{t} g(t-\tau)(\Delta u(\tau), \Delta w) d \tau+\left(\rho\left(u_{t}(t)\right), w\right)=0
\end{aligned}
$$

for all $w \in V$. Let $\left\{w^{j}\right\}$ be a complete orthogonal system of $W$. For each $m \in \mathbb{N}$, let $V_{m}$ be the subspace generated by $\left\{w^{1}, w^{2}, \cdots, w^{m}\right\}$. We search for a function

$$
u^{m}(t)=\sum_{j=1}^{m} \delta_{m}^{j}(t) w^{j}
$$

satisfying the approximate equation

$$
\begin{aligned}
\left(u_{t t}^{m}(t), w\right)+\left(\Delta u^{m}(t),\right. & \Delta w)+M\left(\left\|\nabla u^{m}\right\|^{2}\right)\left(\nabla u^{m}(t), \nabla w\right) \\
& -\int_{0}^{t} g(t-\tau)\left(\Delta u^{m}(\tau), \Delta w\right) d \tau+\left(\rho\left(u_{t}^{m}(t)\right), w\right)=0
\end{aligned}
$$

with initial data

$$
u^{m}(0)=u_{0}^{m} \rightarrow u_{0} \quad \text { in } \quad W \quad \text { and } \quad u_{t}^{m}(0)=u_{1}^{m} \rightarrow u_{1} \quad \text { in } \quad V .
$$

By standard methods in differential equation, we prove the existence of solutions to the approximate equation $(3.1)$ on some interval $\left[0, t_{m}\right)$. Then, this solution can be extended to the whole interval $[0, T]$, where $T=\infty$, by using the following first estimate.

3.1. The first estimate. Replacing $w$ by $u_{t}^{m}(t)$ in equation (3.1) we obtain

$$
\begin{aligned}
& \frac{1}{2} \frac{d}{d t}\left(\left\|u_{t}^{m}(t)\right\|^{2}+\left\|\Delta u^{m}(t)\right\|^{2}+\hat{M}\left(\left\|\nabla u^{m}(t)\right\|^{2}\right)\right) \\
& =\frac{d}{d t}\left(\int_{0}^{t} g(t-\tau)\left(\Delta u^{m}(\tau), \Delta u^{m}(t)\right) d \tau\right) \\
& \quad-\int_{0}^{t} g^{\prime}(t-\tau)\left(\Delta u^{m}(\tau), \Delta u^{m}(t)\right) d \tau \\
& -g(0)\left\|\Delta u^{m}(t)\right\|^{2}-\left(\rho\left(u_{t}^{m}(t)\right), u_{t}^{m}(t)\right)
\end{aligned}
$$


Considering the Cauchy-Schwartz inequality and taking hypotheses of $g$ into account, we deduce

$$
\begin{aligned}
& \left|\int_{0}^{t} g^{\prime}(t-\tau)\left(\Delta u^{m}(\tau), \Delta u^{m}(t)\right) d \tau\right| \\
& \leq\left\|\Delta u^{m}(t)\right\| \int_{0}^{t} \mid g^{\prime}(t-\tau)\|\| \Delta u^{m}(\tau) \| d \tau \\
& \leq \frac{c_{1}^{2}}{2}\left\|\Delta u^{m}(t)\right\|^{2}+\frac{1}{2}\|g\|_{L^{1}(0, \infty)} \int_{0}^{t} g(t-\tau)\left\|\Delta u^{m}(\tau)\right\|^{2} d \tau .
\end{aligned}
$$

From (2.2), (2.7), (3.3) and (3.4), we deduce by integration over $(0, t)$

$$
\begin{aligned}
& \frac{1}{2}\left(\left\|u_{t}^{m}(t)\right\|^{2}+\left\|\Delta u^{m}(t)\right\|^{2}+s_{0}\left\|\nabla u^{m}(t)\right\|^{2}\right) \\
& \leq \frac{1}{2}\left(\left\|u_{1}^{m}\right\|^{2}+\left\|\Delta u_{0}^{m}\right\|^{2}+\hat{M}\left(\left\|\nabla u_{0}^{m}\right\|^{2}\right)+\int_{0}^{t} g(t-\tau)\left(\Delta u^{m}(\tau), \Delta u^{m}(t)\right) d \tau\right. \\
& \quad+\frac{c_{1}^{2}}{2} \int_{0}^{t}\left\|\Delta u^{m}(s)\right\|^{2} d s+\frac{1}{2}\|g\|_{L^{1}(0, \infty)}^{2} \int_{0}^{t}\left\|\Delta u^{m}(s)\right\|^{2} d s .
\end{aligned}
$$

On the other hand, using the inequality $a b \leq \frac{1}{4 \epsilon} a^{2}+\epsilon b^{2}$, we have

$$
\begin{aligned}
\int_{0}^{t} g(t-\tau) & \left(\Delta u^{m}(\tau), \Delta u^{m}(t)\right) d \tau \\
& \leq \epsilon\left\|\Delta u^{m}(t)\right\|^{2}+\frac{1}{4 \epsilon}\|g\|_{L^{1}(0, \infty)}\|g\|_{L^{\infty}(0, \infty)} \int_{0}^{t}\left\|\Delta u^{m}(\tau)\right\|^{2} d \tau .
\end{aligned}
$$

Replacing (3.6) in (3.5) with $\epsilon>0$ sufficiently small and employing Gronwall's lemma we obtain the first estimate

$$
\left\|u_{t}^{m}(t)\right\|^{2}+\left\|\Delta u^{m}(t)\right\|^{2}+\left\|\nabla u^{m}(t)\right\|^{2} \leq C_{1},
$$

where $C_{1}$ is a positive constant. Therefore, the approximate solution $u^{m}(t)$ can be extended to the whole interval $[0, T]$, where $T=\infty$.

3.2. The second estimate. Preliminary to the second estimate, we introduce the useful lemma. The following lemma (with $t=0$ ) will be used to estimate $\left\|u_{t t}^{m}(t)\right\|$.

Lemma 3.1. (cf. [17]) $\left\|\rho\left(\cdot, u_{t}^{m}(t)\right)\right\| \leq C$ with $C=C\left(u_{0}, u_{1}\right)$ a positive constant (independent of $t, m$ ).

In order to estimate $\left\|u_{t t}^{m}(t)\right\|^{2}$, we need to estimate $\left\|u_{t t}^{m}(0)\right\|$.

First of all, we are estimating $u_{t t}^{m}(0)$ in the $L^{2}$-norm. Considering $t=0$ and $w=u_{t t}^{m}(0)$ in (3.1), we obtain

$$
\left\|u_{t t}^{m}(0)\right\|^{2} \leq\left(\left\|\Delta^{2} u_{0}^{m}\right\|+M\left(\left\|\nabla u_{0}^{m}\right\|^{2}\right)\left\|\Delta u_{0}^{m}\right\|+\left\|\rho\left(u_{1}^{m}\right)\right\|\right)\left\|u_{t t}^{m}(0)\right\| .
$$

From the previous lemma and hypotheses on the initial data, it follows that

$$
\left\|u_{t t}^{m}(0)\right\| \leq C_{2} \quad \text { for all } \quad m \in \mathbb{N},
$$

where $C_{2}$ is a positive constant. 
Now we are going to obtain an estimate for $u_{t t}^{m}$ and $\Delta u_{t}^{m}$ in $L^{2}$-norm. Finally, differentiating (3.1) with respect to $t$ and substituting $w=u_{t t}^{m}(t)$, we have

$$
\begin{aligned}
& \frac{1}{2} \frac{d}{d t}\left(\left\|u_{t t}^{m}(t)\right\|^{2}+\left\|\Delta u_{t}^{m}(t)\right\|^{2}\right) \\
& =+2 M^{\prime}\left(\left\|\nabla u^{m}(t)\right\|^{2}\right)\left(\nabla u^{m}(t), \nabla u_{t}^{m}(t)\right)\left(\Delta u^{m}(t), u_{t t}^{m}(t)\right) \\
& +M\left(\left\|\nabla u^{m}(t)\right\|^{2}\right)\left(\Delta u^{m}(t), u_{t t}^{m}(t)\right) \\
& \quad+g(0) \frac{d}{d t}\left(\Delta u^{m}(t), \Delta u_{t}^{m}(t)\right)-g(0)\left\|\Delta u_{t}^{m}(t)\right\|^{2} \\
& +\frac{d}{d t}\left(\int_{0}^{t} g^{\prime}(t-\tau)\left(\Delta u^{m}(\tau), \Delta u_{t}^{m}(t)\right) d \tau\right) \\
& \quad-\int_{0}^{t} g^{\prime \prime}(t-\tau)\left(\Delta u^{m}(\tau), \Delta u_{t}^{m}(t)\right) d \tau-g^{\prime}(0)\left(\Delta u^{m}(t), \Delta u_{t}^{m}(t)\right) \\
& -\left(\frac{\partial \rho(x, s)}{\partial s} u_{t t}^{m}(t), u_{t t}^{m}(t)\right) .
\end{aligned}
$$

Since $M \in C^{1}\left(\mathbb{R}_{+}\right)$and (3.7), using the Young's inequality and Sobolev imbedding theorem we get

(3.10)

$\left|2 M^{\prime}\left(\left\|\nabla u^{m}(t)\right\|^{2}\right)\left(\nabla u^{m}(t), \nabla u_{t}^{m}(t)\right)\left(\Delta u^{m}(t), u_{t t}^{m}(t)\right)\right| \leq d_{1}\left(\left\|\Delta u_{t}^{m}(t)\right\|^{2}+\left\|u_{t t}^{m}(t)\right\|^{2}\right)$, where $d_{1}$ is a positive constant. Similarly, we can easily check that

$$
\left|M\left(\left\|\nabla u^{m}(t)\right\|^{2}\right)\left(\Delta u^{m}(t), u_{t t}^{m}(t)\right)\right| \leq d_{2}\left(\epsilon\left\|\Delta u^{m}(t)\right\|^{2}+\frac{1}{4 \epsilon}\left\|u_{t t}^{m}(t)\right\|^{2}\right),
$$

where $d_{2}$ is a positive constant.

On the other hand, from (2.5), we easily obtain as similar calculation of (3.4)

$$
\begin{aligned}
\mid \int_{0}^{t} g^{\prime \prime}(t-\tau)( & \left.\Delta u^{m}(\tau), \Delta u_{t}^{m}(t)\right) d \tau \mid \\
\leq & \frac{c_{3}^{2}}{4 \epsilon}\left\|\Delta u_{t}^{m}(t)\right\|^{2}+\epsilon\|g\|_{L^{1}(0, \infty)} \int_{0}^{t} g(t-\tau)\left\|\Delta u^{m}(\tau)\right\|^{2} d \tau .
\end{aligned}
$$

Replacing (3.10) - (3.12) in (3.9) and using the positivity of $\frac{\partial \rho}{\partial s}(\mathrm{cf} .(2.7))$, and then integrating $(3.9)$ over $(0, t)$ we have

$$
\begin{aligned}
& \frac{1}{2}\left(\left\|u_{t t}^{m}(t)\right\|^{2}+\left\|\Delta u_{t}^{m}(t)\right\|^{2}\right) \\
& \leq \frac{1}{2}\left\|u_{t t}^{m}(0)\right\|^{2}+\frac{1}{2}\left\|\Delta u_{1}^{m}\right\|^{2}+d_{1} \int_{0}^{t}\left(\left\|u_{t t}^{m}(s)\right\|^{2}+\left\|\Delta u_{t}^{m}(s)\right\|^{2}\right) d s \\
& \quad+\frac{d_{2}}{4 \epsilon} \int_{0}^{t}\left\|u_{t t}^{m}(s)\right\|^{2} d s+d_{2} \epsilon \int_{0}^{t}\left\|\Delta u^{m}(s)\right\|^{2} d s+g(0)\left(\Delta u^{m}(t), \Delta_{t}^{m}(t)\right) \\
& \quad+\frac{c_{3}^{2}}{4 \epsilon} \int_{0}^{t}\left\|\Delta u_{t}^{m}(s)\right\|^{2} d s+\epsilon\|g\|_{L^{1}(0, \infty)}^{2} \int_{0}^{t}\left\|\Delta u^{m}(s)\right\|^{2} d s \\
& \quad+\int_{0}^{t} g^{\prime}(t-\tau)\left(\Delta u^{m}(\tau), \Delta u_{t}^{m}(t)\right) d \tau-\int_{0}^{t} g^{\prime}(0)\left(\Delta u^{m}(s), \Delta u_{t}^{m}(s)\right) d s .
\end{aligned}
$$

We note that

$$
\left|g(0)\left(\Delta u^{m}(t), \Delta u_{t}^{m}(t)\right)\right| \leq \frac{(g(0))^{2}}{4 \epsilon}\left\|\Delta u^{m}(t)\right\|^{2}+\epsilon\left\|\Delta u_{t}^{m}(t)\right\|^{2},
$$




$$
\begin{aligned}
& \left|\int_{0}^{t} g^{\prime}(t-\tau)\left(\Delta u^{m}(\tau), \Delta u_{t}^{m}(t)\right) d \tau\right| \\
& \leq \epsilon\left\|\Delta u_{t}^{m}(t)\right\|^{2}+\frac{c_{1}^{2}}{4 \epsilon}\|g\|_{L^{1}(0, \infty)}\|g\|_{L^{\infty}(0, \infty)} \int_{0}^{t}\left\|\Delta u^{m}(\tau)\right\|^{2} d \tau
\end{aligned}
$$

and

$$
\begin{aligned}
& \left|\int_{0}^{t} g^{\prime}(0)\left(\Delta u^{m}(s), \Delta u_{t}^{m}(s)\right) d s\right| \\
& \leq\left(g^{\prime}(0)\right)^{2} \epsilon \int_{0}^{t}\left\|\Delta u^{m}(s)\right\|^{2} d s+\frac{1}{4 \epsilon} \int_{0}^{t}\left\|\Delta u_{t}^{m}(s)\right\|^{2} d s .
\end{aligned}
$$

Substituting (3.14) - (3.16) in (3.13) with $\epsilon>0$ sufficiently small and taking into account (3.2), (3.7) and (3.8), from Gronwall's lemma we obtain the second estimate

$$
\left\|u_{t t}^{m}(t)\right\|^{2}+\left\|\Delta u_{t}^{m}(t)\right\|^{2} \leq C_{2},
$$

where $C_{2}$ is a positive constant.

By estimates (3.8) and (3.17), we obtain

$$
\begin{gathered}
\left(u^{m}\right) \quad \text { is bounded in } \quad L^{\infty}(0, T ; V), \\
\left(u_{t}^{m}\right) \quad \text { is bounded in } \quad L^{\infty}\left(0, T ; L^{2}(\Omega)\right), \\
\left(u_{t}^{m}\right) \quad \text { is bounded in } \quad L^{\infty}(0, T ; V), \\
\left(u_{t t}^{m}\right) \quad \text { is bounded in } \quad L^{\infty}\left(0, T ; L^{2}(\Omega)\right) .
\end{gathered}
$$

Therefore, we get a subsequence of $\left(u^{m}\right)$, which from now on will be represented by the same notation, such that

$$
\begin{gathered}
u^{m} \rightarrow u \quad \text { weak star in } \quad L^{\infty}(0, T ; V), \\
u_{t}^{m} \rightarrow u_{t} \quad \text { weak star in } \quad L^{\infty}\left(0, T ; L^{2}(\Omega)\right), \\
u_{t}^{m} \rightarrow u_{t} \quad \text { weak star in } \quad L^{\infty}(0, T ; V), \\
u_{t t}^{m} \rightarrow u_{t t} \quad \text { weak star in } \quad L^{\infty}\left(0, T ; L^{2}(\Omega)\right) .
\end{gathered}
$$

From Aubin-Lions lemma, we deduce that

$$
\begin{gathered}
u^{m} \rightarrow u \quad \text { strongly in } \quad C([0, T] ; V), \\
u_{t}^{m} \rightarrow u_{t} \quad \text { strongly in } \quad C\left([0, T] ; L^{2}(\Omega)\right) .
\end{gathered}
$$

The above convergences (3.18) - (3.23) and the fact that $\left(\rho\left(x, u_{t}^{m}\right), v\right) \rightarrow\left(\rho\left(x, u_{t}\right), v\right)$ in $D^{\prime}(0, T)$, for all $v \in V$ (cf. Lemma 4.4. in [17]) are enough to pass to the limit in (3.1). Then it is a matter of routine to conclude the existence of global solutions in $[0, T]$. 
3.3. Uniqueness. Let $u^{1}$ and $u^{I}$ be two solutions to problem (1.1). Then, $z:=u^{1}-u^{I}$ verifies

$$
\begin{aligned}
&\left(z_{t t}(t), w\right)+(\Delta z(t), \Delta w)+\left(\rho\left(u_{t}^{1}(t)\right), w\right)-\left(\rho\left(u_{t}^{I}(t)\right), w\right) \\
&=\int_{0}^{t} g(t-\tau)(\Delta z(\tau), \Delta w) d \tau+M\left(\left\|\nabla u^{I}\right\|^{2}\right)\left(\nabla u^{I}(t), \nabla w\right) \\
&-M\left(\left\|\nabla u^{1}\right\|^{2}\right)\left(\nabla u^{1}(t), \nabla w\right)
\end{aligned}
$$

for all $w \in V$. Replacing $w=z_{t}(t)$ in (3.24) and adding the term

$$
M\left(\left\|\nabla u^{I}\right\|^{2}\right)\left(\nabla u^{1}(t), \nabla z_{t}(t)\right)
$$

both sides of (3.24), it follows that

$$
\begin{aligned}
\frac{1}{2} \frac{d}{d t}\left(\left\|z_{t}(t)\right\|^{2}+\right. & \left.\|\Delta z(t)\|^{2}\right)+M\left(\left\|\nabla u^{I}\right\|^{2}\right)\left(\nabla z(t), \nabla z_{t}(t)\right) \\
& +\left(\rho\left(u_{t}^{1}(t)\right)-\rho\left(u_{t}^{I}(t)\right), z_{t}(t)\right) \\
= & \int_{0}^{t} g(t-\tau)\left(\Delta z(\tau), \Delta z_{t}(t)\right) d \tau+ \\
& \left(M\left(\left\|\nabla u^{1}\right\|^{2}\right)-M\left(\left\|\nabla u^{I}\right\|^{2}\right)\right)\left(\Delta u^{1}(t), z_{t}(t)\right) .
\end{aligned}
$$

On the other hand, we note that

$$
\begin{aligned}
& \frac{d}{d t}\left[M\left(\left\|\nabla u^{I}\right\|^{2}\right)\|\nabla z(t)\|^{2}\right] \\
& \quad=2 M^{\prime}\left(\left\|\nabla u^{I}\right\|^{2}\right)\left(\nabla u^{I}(t), \nabla u_{t}^{I}(t)\right)\|\nabla z(t)\|^{2}+2 M\left(\left\|\nabla u^{I}\right\|^{2}\right)\left(\nabla z(t), \nabla z_{t}(t)\right) .
\end{aligned}
$$

Replacing above equality in (3.25), we get

$$
\begin{aligned}
& \frac{1}{2} \frac{d}{d t}\left(\left\|z_{t}(t)\right\|^{2}+\|\Delta z(t)\|^{2}+M\left(\left\|\nabla u^{I}\right\|^{2}\right)\|\nabla z(t)\|^{2}\right) \\
& +\left(\rho\left(u_{t}^{1}(t)\right)-\rho\left(u_{t}^{I}(t)\right), z_{t}(t)\right) \\
& =\int_{0}^{t} g(t-\tau)\left(\Delta z(\tau), \Delta z_{t}(t)\right) d \tau+\left(M\left(\left\|\nabla u^{1}\right\|^{2}\right)\right. \\
& \left.-M\left(\left\|\nabla u^{I}\right\|^{2}\right)\right)\left(\Delta u^{1}(t), z_{t}(t)\right) \\
& \quad+M^{\prime}\left(\left\|\nabla u^{I}\right\|^{2}\right)\left(\nabla u^{I}(t), \nabla u_{t}^{I}(t)\right)\|\nabla z(t)\|^{2} .
\end{aligned}
$$

We observe that

$$
\begin{aligned}
& \int_{0}^{t} g(t-\tau)\left(\Delta z(\tau), \Delta z_{t}(t)\right) d \tau \\
&=-g(0)\|\Delta z(t)\|^{2}-\int_{0}^{t} g^{\prime}(t-\tau)(\Delta z(\tau), \Delta z(t)) d \tau+ \\
& \frac{d}{d t}\left(\int_{0}^{t} g(t-\tau)(\Delta z(\tau), \Delta z(t)) d \tau\right),
\end{aligned}
$$




$$
\begin{aligned}
& \left|M\left(\left\|\nabla u^{1}\right\|^{2}\right)-M\left(\left\|\nabla u^{I}\right\|^{2}\right)\right| \\
& =\left|\int_{\left\|\nabla u^{I}\right\|^{2}}^{\left\|\nabla u^{1}\right\|^{2}} M^{\prime}(s) d s\right| \leq d_{3}\left|\left\|\nabla u^{1}\right\|^{2}-\left\|\nabla u^{I}\right\|^{2}\right| \leq d_{4}|| \nabla z(t) \|,
\end{aligned}
$$

and

$$
M^{\prime}\left(\left\|\nabla u^{I}\right\|^{2}\right)\left(\nabla u^{I}(t), \nabla u_{t}^{I}(t)\right)\|\nabla z(t)\|^{2} \leq d_{5}\left\|\nabla u_{t}^{I}(t)\right\|\|\nabla z(t)\|^{2},
$$

where $d_{3}, d_{4}$ and $d_{5}$ are positive constants.

By continuity of $\rho(\cdot, s)$ and $\frac{\partial \rho}{\partial s}$ and the mean value theorem for vector-valued differential functions, we conclude that

$$
\left(\rho\left(u_{t}^{1}(t)\right)-\rho\left(u_{t}^{I}(t)\right), z_{t}(t)\right)=\left(\frac{\partial \rho(\bar{s})}{\partial s} z_{t}(t), z_{t}(t)\right),
$$

for some $\bar{s}$ in the line between $u_{t}^{1}$ and $u_{t}^{I}$.

Replacing (3.27)-(3.30) in (3.26) and using the positivity of $\frac{\partial \rho}{\partial s}$ and the first and second estimate, we arrive that

$$
\begin{aligned}
& \frac{1}{2} \frac{d}{d t}\left(\left\|z_{t}(t)\right\|^{2}+\|\Delta z(t)\|^{2}+M\left(\left\|\nabla u^{I}\right\|^{2}\right)\|\nabla z(t)\|^{2}\right) \\
& \leq \frac{c_{1}^{2}}{2}\|\Delta z(t)\|^{2}+\frac{1}{2}\|g\|_{L^{1}(0, \infty)} \int_{0}^{t} g(t-\tau)\|\Delta z(\tau)\|^{2} d \tau \\
& \quad+\frac{d}{d t}\left(\int_{0}^{t} g(t-\tau)(\Delta z(\tau), \Delta z(t)) d \tau\right)+d_{6}\left\|z_{t}(t)\right\|^{2}+d_{7}\|\nabla z(t)\|^{2},
\end{aligned}
$$

where $d_{6}$ and $d_{7}$ are positive constants.

Now, integrating $(3.31)$ over $(0, t)$ and noting that

$\int_{0}^{t} g(t-\tau)(\Delta z(\tau), \Delta z(t)) d \tau \leq \epsilon\|\Delta z(t)\|^{2}+\frac{1}{4 \epsilon}\|g\|_{L^{1}(0, \infty)}\|g\|_{L^{\infty}(0, \infty)} \int_{0}^{t}\|\Delta z(\tau)\|^{2} d \tau$.

Then, we conclude by choosing $\epsilon>0$ sufficiently small and employing Gronwall's lemma $\left\|z_{t}(t)\right\|=\|\nabla z(t)\|=\|\Delta z(t)\|=0$.

3.4. Weak solutions. Let us $\left\{u_{0}, u_{1}\right\} \in V \times L^{2}(\Omega)$. Then, by density, there exists $\left\{u_{0}^{m}, u_{1}^{m}\right\} \subset W \times V$ such that

$$
u_{0}^{m} \rightarrow u_{0} \quad \text { in } \quad V \quad \text { and } \quad u_{1}^{m} \rightarrow u_{1} \quad \text { in } \quad L^{2}(\Omega) .
$$

Therefore, for each $m \in \mathbb{N}$, there exists $u^{m}$, smooth solution of problem (1.1) verifying

$$
\left\{\begin{array}{l}
u_{t t}^{m}+\Delta^{2} u^{m}-M\left(\| \nabla u^{m}||^{2}\right) \Delta u^{m}-\int_{0}^{t} g(t-\tau) \Delta^{2} u^{m}(\tau) d \tau+\rho\left(x, u_{t}^{m}\right)=0 \\
u^{m}(0)=u_{0}^{m}, \quad u_{t}^{m}(0)=u_{1}^{m} .
\end{array}\right.
$$

Repeating the same argument used in the first estimate, we obtain

$$
\left\|u_{t}^{m}(t)\right\|^{2}+\left\|\Delta u^{m}(t)\right\|^{2}+\left\|\nabla u^{m}(t)\right\|^{2} \leq C_{3},
$$

where $C_{3}$ is a positive constant.

Let $z^{m, l}=u^{m}-u^{l}$ with $m, l \in \mathbb{N}$, where $u^{m}$ and $u^{l}$ are regula solutions of (3.33). Then following the same already used in the uniqueness of regular solutions and taking the (3.32) into account, we deduce that there exists $u$ such that

$$
u^{m} \rightarrow u \quad \text { strongly in } \quad C([0, T] ; V),
$$




$$
u_{t}^{m} \rightarrow u_{t} \quad \text { strongly in } \quad C\left([0, T] ; L^{2}(\Omega)\right) .
$$

From (3.34) - (3.36), we can pass to the limit using standard arguments in order to obtain

$$
u_{t t}+\Delta^{2} u-M\left(\|\nabla u\|^{2}\right) \Delta u-\int_{0}^{t} g(t-\tau) \Delta^{2} u(\tau) d \tau+\rho\left(x, u_{t}\right)=0
$$

in $L^{2}\left(0, \infty, V^{\prime}\right)$, where $V^{\prime}$ is a dual space of $V$. The uniqueness of weak solutions can be also obtained by same argument of subsection 3.3 .

\section{Energy decay}

In this section we prove the energy decay rate to problem (1.1) using the lemma 2.2. It is enough to consider $u_{0} \in W \cap V, u_{1} \in V$ and then to use a density argument.

We define the energy $E(t)$ of the problem (1.1) by

$$
E(t)=\frac{1}{2} \int_{\Omega}\left|u^{\prime}\right|^{2} d x+\frac{1}{2} \int_{\Omega}|\Delta u|^{2} d x+\frac{1}{2} \hat{M}\left(\|\left.\nabla u\right|^{2}\right) .
$$

Then the derivative of the energy is given by

$$
E^{\prime}(t)=-\left(\rho\left(x, u^{\prime}\right), u^{\prime}\right)+\int_{0}^{t} g(t-\tau)\left(\Delta u(\tau), \Delta u^{\prime}(t)\right) d \tau
$$

Defining

$$
(g \square \Delta u)(t)=\int_{0}^{t} g(t-\tau)\|\Delta u(\tau)-\Delta u(t)\|^{2} d \tau .
$$

A direct computation shows that

$$
\begin{aligned}
& \int_{0}^{t} g(t-\tau)\left(\Delta u(\tau), \Delta u^{\prime}(t)\right) d \tau=\frac{1}{2}\left(g^{\prime} \square \Delta u\right)(t)-\frac{1}{2}(g \square \Delta u)^{\prime}(t) \\
& +\frac{1}{2} \frac{d}{d t}\left\{\int_{0}^{t} g(t-\tau) d \tau\|\Delta u\|^{2}\right\}-\frac{1}{2} g(t)\|\Delta u\|^{2} .
\end{aligned}
$$

We define the modified energy by

$$
\begin{aligned}
e(t)= & \frac{1}{2} \int_{\Omega}\left|u^{\prime}\right|^{2} d x+\frac{1}{2}\left(1-\int_{0}^{t} g(t-\tau) d \tau\right) \int_{\Omega}|\Delta u|^{2} d x+ \\
& \frac{1}{2} \hat{M}\left(\|\nabla u\|^{2}\right)+\frac{1}{2}(g \square \Delta u)(t) .
\end{aligned}
$$

Then

$$
e^{\prime}(t)=-\left(\rho\left(x, u^{\prime}\right), u^{\prime}\right)+\frac{1}{2}\left(g^{\prime} \square \Delta u\right)(t)-\frac{1}{2} g(t)\|\Delta u\|^{2} .
$$

We observe that in view of assumption (2.3) we have $e(t) \geq 0$, and according to hypotheses on $g$ we deduce that $e^{\prime}(t) \leq 0$. Moreover,

$$
E(t) \leq \ell^{-1} e(t) \quad \text { for all } \quad t \geq 0 .
$$

Therefore, it is enough to obtain the decay for the modified energy $e(t)$.

Firstly, in order to prove the decay of $e(t)$ we introduce useful properties. 
4.1. Some identities and the basic inequalities. Let $u$ be the solution of (1.1) and $T>0$ fixed.

Firstly, multiplying the equation by $u^{\prime}$ and integrating over $[t, t+T] \times \Omega$, we have

$$
\begin{aligned}
& \int_{t}^{t+T} \int_{\Omega} \rho\left(x, u^{\prime}\right) u^{\prime} d x d s-\frac{1}{2} \int_{t}^{t+T}\left(g^{\prime} \square \Delta u\right)(s) d s \\
& +\frac{1}{2} \int_{t}^{t+T} g(s)\|\Delta u\|^{2} d s=e(t)-e(t+T) .
\end{aligned}
$$

Second, multiplying the equation by $u$ and integrating we have

$$
\begin{aligned}
& \int_{t}^{t+T} \int_{\Omega}\left(-\left|u^{\prime}\right|^{2}+|\Delta u|^{2}\right) d x d s+\int_{t}^{t+T} M\left(|| \nabla u \|^{2}\right)|| \nabla u \|^{2} d s \\
& -\int_{t}^{t+T} \int_{0}^{s} g(s-\tau)(\Delta u(\tau), \Delta u(s)) d \tau d s \\
& =-\int_{t}^{t+T} \int_{\Omega} \rho\left(x, u^{\prime}\right) u d x d s+\left(u^{\prime}(t), u(t)\right)-\left(u^{\prime}(t+T), u(t+T)\right) .
\end{aligned}
$$

Third, multiplying the equation by $m(x) \cdot \nabla u$ we have

$$
\begin{aligned}
& \frac{n}{2} \int_{t}^{t+T} \int_{\Omega}\left|u^{\prime}\right|^{2} d x d s+\left(2-\frac{n}{2}\right) \int_{t}^{t+T} \int_{\Omega}|\Delta u|^{2} d x d s \\
& +\left(1-\frac{n}{2}\right) \int_{t}^{t+T} M\left(\|\nabla u\|^{2}\right)\|\nabla u\|^{2} d s \\
& \quad-2 \int_{t}^{t+T} \int_{0}^{s} g(s-\tau)(\Delta u(\tau), \Delta u(s)) d \tau d s \\
& -\int_{t}^{t+T} \int_{0}^{s} g(s-\tau)(\Delta u(\tau), m \cdot \nabla(\Delta u(s))) d \tau d s \\
& \quad+\int_{t}^{t+T} \int_{0}^{s} g(s-\tau) \int_{\Gamma}(m \cdot \nu) \Delta u(\tau) \Delta u(s) d \Gamma d \tau d s \\
& +\int_{t}^{t+T} \int_{\Omega} \rho\left(x, u^{\prime}\right)(m(x) \cdot \nabla u) d x d s \\
& =\left(u^{\prime}(t), m(x) \cdot \nabla u(t)\right)-\left(u^{\prime}(t+T), m(x) \cdot \nabla u(t+T)\right) \\
& +\frac{1}{2} \int_{t}^{t+T} \int_{\Gamma}(m \cdot \nu)|\Delta u|^{2} d \Gamma d s .
\end{aligned}
$$

Next, take a function $\zeta \in W^{2, \infty}(\Omega)$ such that $\frac{|\nabla \zeta|^{2}}{\zeta}$ and $\frac{|\Delta \zeta|^{2}}{\zeta}$ are bounded and

$$
0 \leq \zeta(x) \leq 1 \quad \text { in } \Omega, \quad \zeta=1 \text { in } \tilde{\omega} \text { and } \zeta=0 \text { in } \bar{\Omega} \backslash \omega
$$


where $\tilde{\omega}$ is an open set in $\bar{\Omega}$ with $\Gamma_{1} \subset \tilde{\omega} \subset \omega \subset \bar{\Omega}$ (cf. [11]). Then, multiplying the equation $\zeta u$ and integrating we have

$$
\begin{aligned}
& \int_{t}^{t+T} \int_{\Omega} \zeta\left(|\Delta u|^{2}-\left|u^{\prime}\right|^{2}+M\left(|| \nabla u||^{2}\right)|\nabla u|^{2}\right) d x d s \\
& =\left(u^{\prime}(t), \zeta u(t)\right)-\left(u^{\prime}(t+T), \zeta u(t+T)\right) \\
& -\int_{t}^{t+T} \int_{\Omega}(\Delta u \Delta \zeta u+2 \Delta u(\nabla \zeta \cdot \nabla u)) d x d s \\
& \quad-\int_{t}^{t+T} M\left(\left.|| \nabla u\right|^{2}\right) \int_{\Omega} u(\nabla u \cdot \nabla \zeta) d x d s \\
& -\int_{t}^{t+T} \int_{\Omega} \rho\left(x, u^{\prime}\right) \zeta u d x d s \\
& \quad+\int_{t}^{t+T} \int_{0}^{s} g(s-\tau)(\Delta u(\tau), \Delta \zeta u(s) \\
& +2 \nabla \zeta \cdot \nabla u(s)+\zeta \Delta u(s)) d \tau d s .
\end{aligned}
$$

Finally, take a vector field $h=\left(h^{1}, h^{2}, \cdots, h^{n}\right): \bar{\Omega} \rightarrow \mathbb{R}^{n}$ of $C^{2}$ class such that

$$
h=\nu \text { on } \Gamma_{1}, \quad h \cdot \nu \geq 0 \text { on } \Gamma \text { and } h=0 \text { in } \Omega \backslash \hat{\omega},
$$

where $\hat{\omega}$ is and open set in $\mathbb{R}^{n}$ with $\Gamma_{1} \subset \hat{\omega} \cap \bar{\Omega} \subset \omega$ (cf. [11]). Then, multiplying the equation by $h \cdot \nabla u$ and integrating we have

$$
\begin{aligned}
& \frac{1}{2} \int_{t}^{t+T} \int_{\Gamma}(h \cdot \nu)|\Delta u|^{2} d \Gamma d s \\
& =\frac{1}{2} \int_{t}^{t+T} \int_{\Omega} \operatorname{divh}\left(\left|u^{\prime}\right|^{2}-|\Delta u|^{2}-M\left(\left.|| \nabla u\right|^{2}\right)|\nabla u|^{2}\right) d x d s \\
& +\int_{t}^{t+T} \int_{\Omega}(\Delta h \cdot \nabla u) \Delta u d x d s \\
& \quad+2 \int_{t}^{t+T} \int_{\Omega} \Delta u \sum_{i, j=1}^{n} \frac{\partial h^{j}}{\partial x_{i}}\left(\frac{\partial}{\partial x_{i}} \frac{\partial u}{\partial x_{j}}\right) d x d s \\
& +\int_{t}^{t+T} M\left(|| \nabla u \|^{2}\right) \int_{\Omega} \sum_{i, j=1}^{n} \frac{\partial h^{j}}{\partial x_{i}} \frac{\partial u}{\partial x_{i}} \frac{\partial u}{\partial x_{j}} d x d s \\
& \quad-\int_{t}^{t+T} \int_{0}^{s} g(s-\tau)(\Delta u(\tau), \Delta h \cdot \nabla u(s)) d \tau d s \\
& \quad-\int_{t}^{t+T} \int_{0}^{s} g(s-\tau) \int_{\Omega} \Delta u(\tau) \sum_{i, j=1}^{n} \frac{\partial h^{j}}{\partial x_{i}}\left(\frac{\partial}{\partial x_{i}} \frac{\partial u(s)}{\partial x_{j}}\right) d x d \tau d s
\end{aligned}
$$




$$
\begin{aligned}
& +\int_{t}^{t+T} \int_{0}^{s} g(s-\tau) \int_{\Gamma}(h \cdot \nu) \Delta u(\tau) \Delta u(s) d \Gamma d \tau d s \\
& -\int_{t}^{t+T} \int_{0}^{s} g(s-\tau)(\Delta u(\tau), \nabla h \cdot \Delta u(s)) d \tau d s \\
& -\int_{t}^{t+T} \int_{0}^{s} g(s-\tau)(\Delta u(\tau), h \cdot \nabla(\Delta u(s))) d \tau d s \\
& \quad+\left(u^{\prime}(t+T), h \cdot \nabla u(t+T)\right)-\left(u^{\prime}(t), h \cdot \nabla u(t)\right) \\
& +\int_{t}^{t+T} \int_{\Omega} \rho\left(x, u^{\prime}\right)(h \cdot \nabla u) d x d s .
\end{aligned}
$$

Now, our basic inequalities read as follows. And in the section the symbol $C$ indicates positive constants, which may be different.

Proposition 4.1. There exists a fixed $T>0$ such that the modified energy $e(t)$ satisfies

$$
\begin{aligned}
e(t) \leq C\left\{e(t)-e(t+T)+\int_{t}^{t+T}\right. & \int_{\Omega}\left|\rho\left(x, u^{\prime}\right)\right|(|u|+|\nabla u|) d x d s \\
& \left.+\int_{t}^{t+T} \int_{\omega}\left(\left|u^{\prime}\right|^{2}+|u|^{2}+|\nabla u|^{2}\right) d x d s\right\},
\end{aligned}
$$

for all $t>0$.

Proof. Let $\beta$ be a positive number such that $\frac{n \beta}{2}-1>0$. If $n \geq 4$, we also take $\beta$ such that $(n-2) \beta<2$. Then multiplying (4.5) by $\beta$ and adding (4.4) we have

$$
\begin{aligned}
\begin{array}{l}
\left(\frac{n \beta}{2}-1\right) \int_{t}^{t+T} \int_{\Omega}\left|u^{\prime}\right|^{2} d x d s+\left(\beta\left(2-\frac{n}{2}\right)+1\right) \int_{t}^{t+T} \int_{\Omega}|\Delta u|^{2} d x d s \\
\quad+\left(\beta\left(1-\frac{n}{2}\right)+1\right) \int_{t}^{t+T} M\left(\|\left.\nabla u\right|^{2}\right)|| \nabla u \|^{2} d s
\end{array} \\
\underbrace{-(2 \beta+1) \int_{t}^{t+T} \int_{0}^{s} g(s-\tau)(\Delta u(\tau), \Delta u(s)) d \tau d s}_{:=I_{1}} \\
\quad \underbrace{-\beta \int_{t}^{t+T} \int_{0}^{s} g(s-\tau)(\Delta u(\tau), m \cdot \nabla(\Delta u(s))) d \tau d s}_{:=I_{2}} \\
+\beta \int_{t}^{t+T} \int_{0}^{s} g(s-\tau) \int_{\Gamma}(m \cdot \nu) \Delta u(\tau) \Delta u(s) d \Gamma d \tau d s \\
-\left(u^{\prime}(t), \beta(m(x) \cdot \nabla u(t))+u(t)\right) \\
\left.\quad-\int_{t}^{\prime}(t+T), \beta(m(x) \cdot \nabla u(t+T))+u(t+T)\right) \\
+\frac{\beta}{2} \int_{t}^{t+T} \int_{\Omega}^{t+T}[\beta(m(x) \cdot \nabla u)+u] \rho\left(x, u^{\prime}\right) d x d s
\end{aligned}
$$

Now we will estimate $I_{1}$ and $I_{2}$. 
Estimates for $I_{1}:=-(2 \beta+1) \int_{t}^{t+T} \int_{0}^{s} g(s-\tau)(\Delta u(\tau), \Delta u(s)) d \tau d s ;$

Similarly to (3.7) and using Young's inequality, we have

$$
\begin{aligned}
& \int_{0}^{s} g(s-\tau)(\Delta u(\tau), \Delta u(s)) d \tau \\
& =\int_{0}^{s} g(s-\tau)(\Delta u(\tau)-\Delta u(s), \Delta u(s)) d \tau+\int_{0}^{s} g(s-\tau) d \tau \int_{\Omega}|\Delta u|^{2} d x \\
& \leq \frac{1}{2} \int_{\Omega}|\Delta u|^{2} d x+\left.\frac{1}{2}|| g\right|_{L^{1}(0, \infty)}(g \square \Delta u)(s)+\int_{0}^{s} g(s-\tau) d \tau \int_{\Omega}|\Delta u|^{2} d x .
\end{aligned}
$$

Hence, we obtain

$$
\begin{aligned}
& -(2 \beta+1) \int_{t}^{t+T} \int_{0}^{s} g(s-\tau)(\Delta u(\tau), \Delta u(s)) d \tau d s \\
& \geq-(2 \beta+1)\left\{\frac{1}{2} \int_{t}^{t+T} \int_{\Omega}|\Delta u|^{2} d x d s\right. \\
& +\frac{1}{2}\|g\|_{L^{1}(0, \infty)} \int_{t}^{t+T}(g \square \Delta u)(s) d s \\
& \left.+\int_{t}^{t+T} \int_{0}^{s} g(s-\tau) d \tau \int_{\Omega}|\Delta u|^{2} d x d s\right\} .
\end{aligned}
$$

Estimates for $I_{2}:=-\beta \int_{t}^{t+T} \int_{0}^{s} g(s-\tau)(\Delta u(\tau), m \cdot \nabla(\Delta u(s))) d \tau d s ;$

Similarly to $I_{1}$, we have

$$
\begin{aligned}
& \int_{0}^{s} g(s-\tau)(\Delta u(\tau), m \cdot \nabla(\Delta u(s))) d \tau \\
& =\int_{0}^{s} g(s-\tau)(\Delta u(\tau)-\Delta u(s), m \cdot \nabla(\Delta u(s))) d \tau \\
& +\int_{0}^{s} g(s-\tau)(\Delta u(s), m \cdot \nabla(\Delta u(s))) d \tau \\
& \leq \epsilon \int_{\Omega}|\nabla(\Delta u)|^{2} d x+C(\epsilon)\|g\|_{L^{1}(0, \infty)}(g \square \Delta u)(s) \\
& \quad+\frac{1}{2} \int_{0}^{s} g(s-\tau) d \tau \int_{\Gamma}(m \cdot \nu)|\Delta u|^{2} d \Gamma-\frac{n}{2} \int_{0}^{s} g(s-\tau) d \tau \int_{\Omega}|\Delta u|^{2} d x .
\end{aligned}
$$

Hence,taking $\epsilon>0$ sufficiently small, we obtain

$$
\begin{gathered}
-\beta \int_{t}^{t+T} \int_{0}^{s} g(s-\tau)(\Delta u(\tau), m \cdot \nabla(\Delta u(s))) d \tau d s \\
\geq-\beta\left\{\frac{1}{2} \int_{t}^{t+T} \int_{0}^{s} g(s-\tau) d \tau \int_{\Gamma}(m \cdot \nu)|\Delta u|^{2} d \Gamma d s\right. \\
-\frac{n}{2} \int_{t}^{t+T} \int_{0}^{s} g(s-\tau) d \tau \int_{\Omega}|\Delta u|^{2} d x d s \\
\left.\quad+C\|g\|_{L^{1}(0, \infty)} \int_{t}^{t+T}(g \square \Delta u)(s) d s\right\} .
\end{gathered}
$$


Replacing (4.12) and (4.13) in (4.11) and nondecreasing property of $M$, we get

$$
\begin{aligned}
& \left(\frac{n \beta}{2}-1\right) \int_{t}^{t+T} \int_{\Omega}\left|u^{\prime}\right|^{2} d x d s+\left(\left(1-\frac{n}{2}\right) \beta+\frac{1}{2}\right) \\
& \int_{t}^{t+T} \int_{\Omega}\left(1-\int_{0}^{s} g(s-\tau) d \tau\right)|\Delta u|^{2} d x d s \\
& \quad+\left(\left(1-\frac{n}{2}\right) \beta+1\right) \hat{M}\left(\|\nabla u\|^{2}\right)+\frac{1}{2}\|g\|_{L^{1}(0, \infty)} \int_{t}^{t+T}(g \square \Delta u)(s) d s \\
& \leq\left(u^{\prime}(t), \beta(m(x) \cdot \nabla u(t))+u(t)\right) \\
& -\left(u^{\prime}(t+T), \beta(m(x) \cdot \nabla u(t+T))+u(t+T)\right) \\
& \quad-\int_{t}^{t+T} \int_{\Omega}[\beta(m(x) \cdot \nabla u)+u] \rho\left(x, u^{\prime}\right) d x d s \\
& -\beta \int_{t}^{t+T} \int_{0}^{s} g(s-\tau) \int_{\Gamma}(m \cdot \nu) \Delta u(\tau) \Delta u(s) d \Gamma d \tau d s \\
& \quad+\left(\frac{3 \beta}{2}+C+\frac{1}{2}\right)|| g \|_{L^{1}(0, \infty)} \int_{t}^{t+T}(g \square \Delta u)(s) d s \\
& +\beta \int_{t}^{t+T} \int_{\Gamma_{1}}(m \cdot \nu)|\Delta u|^{2} d \Gamma d s .
\end{aligned}
$$

Choosing $\gamma=\min \left\{2\left(\frac{n \beta}{2}-1\right), 2\left(\left(1-\frac{n}{2}\right) \beta+\frac{1}{2}\right),\|g\|_{L^{1}(0, \infty)}\right\}$ and using Poincare's inequality, we arrive that

$$
\begin{aligned}
& \gamma \int_{t}^{t+T} e(s) d s \\
& \leq C(e(t)+e(t+T))+\int_{t}^{t+T} \int_{\Omega}(\beta R|\nabla u|+|u|)\left|\rho\left(x, u^{\prime}\right)\right| d x d s \\
& \underbrace{-\beta \int_{t}^{t+T} \int_{0}^{s} g(s-\tau) \int_{\Gamma}(m \cdot \nu) \Delta u(\tau) \Delta u(s) d \Gamma d \tau d s}_{:=I_{3}} \\
& +\underbrace{+\left(\frac{3 \beta}{2}+C+\frac{1}{2}\right)\|g\|_{L^{1}(0, \infty)} \int_{t}^{t+T}(g \square \Delta u)(s) d s}_{:=I_{4}} \\
& +\beta \int_{t}^{t+T} \int_{\Gamma_{1}}(m \cdot \nu)|\Delta u|^{2} d \Gamma d s .
\end{aligned}
$$

Using Young's inequality and from the fact $m \cdot \nu>0$ on $\Gamma_{1}$, we obtain

$$
\begin{aligned}
& \int_{0}^{s} g(s-\tau) \int_{\Gamma}(m \cdot \nu) \Delta u(\tau) \Delta u(s) d \Gamma d \tau \\
& \leq R \epsilon \int_{0}^{s} g(s-\tau) \int_{\Gamma_{1}}|\Delta u(\tau)|^{2} d \Gamma d \tau \\
& \quad+C(\epsilon) \int_{0}^{s} g(s-\tau) d \tau \int_{\Gamma_{1}}(m \cdot \nu)|\Delta u|^{2} d \Gamma .
\end{aligned}
$$


Hence,

$$
\begin{aligned}
& \left|I_{3}\right| \leq \epsilon R \beta \int_{t}^{t+T} \int_{0}^{s} g(s-\tau) \int_{\Gamma_{1}}|\Delta u(\tau)|^{2} d \Gamma d \tau d s \\
& +C(\epsilon) \beta(1-\ell) \int_{t}^{t+T} \int_{\Gamma_{1}}(m \cdot \nu)|\Delta u|^{2} d \Gamma d s .
\end{aligned}
$$

By definition of $e(t)$, we can easily check that

$$
I_{4} \leq C e(t)
$$

where $C$ is a positive constant that depend on $\beta,\|g\|_{L^{1}(0, \infty)}$ and $T$.

Replacing (4.15) and (4.16) in (4.14) with $\epsilon>0$ sufficiently small, we get (4.17)

$$
\begin{aligned}
\gamma \int_{t}^{t+T} e(s) d s \leq & C(e(t)+e(t+T))+\int_{t}^{t+T} \int_{\Omega}(\beta R|\nabla u|+|u|)\left|\rho\left(x, u^{\prime}\right)\right| d x d s \\
& +C \int_{t}^{t+T} \int_{\Gamma_{1}}(m \cdot \nu)|\Delta u|^{2} d \Gamma d s
\end{aligned}
$$

Next, we shall estimate the last term in (4.17). Since (4.8) and (4.9), the following holds.

$$
\begin{aligned}
& \frac{1}{2} \int_{t}^{t+T} \int_{\Gamma_{1}}|\Delta u|^{2} d \Gamma d s \leq \frac{1}{2} \int_{t}^{t+T} \int_{\Gamma}(h \cdot \nu)|\Delta u|^{2} d \Gamma d s \\
& =\left(u^{\prime}(t+T), h \cdot \nabla u(t+T)\right)-\left(u^{\prime}(t), h \cdot \nabla u(t)\right) \\
& +\int_{t}^{t+T} \int_{0}^{s} g(s-\tau) \int_{\Gamma}(h \cdot \nu) \Delta u(\tau) \Delta u(s) d \Gamma d \tau d s \\
& \quad+\int_{t}^{t+T} \int_{\Omega} \rho\left(x, u^{\prime}\right)(h \cdot \nabla u) d x d s \\
& +\frac{1}{2} \int_{t}^{t+T} \int_{\Omega} d i v h\left(\left|u^{\prime}\right|^{2}-|\Delta u|^{2}-M\left(\|\left.\nabla u\right|^{2}\right)|\nabla u|^{2}\right) d x d s \\
& \quad+\int_{t}^{t+T} \int_{\Omega}(\Delta h \cdot \nabla u) \Delta u d x d s \\
& +2 \int_{t}^{t+T} \int_{\Omega} \Delta u \sum_{i, j=1}^{n} \frac{\partial h^{j}}{\partial x_{i}}\left(\frac{\partial}{\partial x_{i}} \frac{\partial u}{\partial x_{j}}\right) d x d s
\end{aligned}
$$




$$
\begin{aligned}
& +\int_{t}^{t+T} M\left(\|\nabla u\|^{2}\right) \int_{\Omega} \sum_{i, j=1}^{n} \frac{\partial h^{j}}{\partial x_{i}} \frac{\partial u}{\partial x_{i}} \frac{\partial u}{\partial x_{j}} d x d s \\
& -\int_{t}^{t+T} \int_{0}^{s} g(s-\tau)(\Delta u(\tau), \Delta h \cdot \nabla u(s)) d \tau d s \\
& \quad-\int_{t}^{t+T} \int_{0}^{s} g(s-\tau) \int_{\Omega} \Delta u(\tau) \sum_{i, j=1}^{n} \frac{\partial h^{j}}{\partial x_{i}}\left(\frac{\partial}{\partial x_{i}} \frac{\partial u(s)}{\partial x_{j}}\right) d x d \tau d s \\
& \quad-\int_{t}^{t+T} \int_{0}^{s} g(s-\tau)(\Delta u(\tau), \nabla h \cdot \Delta u(s)) d \tau d s \\
& -\int_{t}^{t+T} \int_{0}^{s} g(s-\tau)(\Delta u(\tau), h \cdot \nabla(\Delta u(s))) d \tau d s \\
& :=\left(u^{\prime}(t+T), h \cdot \nabla u(t+T)\right)-\left(u^{\prime}(t), h \cdot \nabla u(t)\right) \\
& \quad+I_{5}+I_{6}+I_{7}+I_{8}+I_{9}+I_{10}-I_{11}-I_{12}-I_{13}-I_{14} .
\end{aligned}
$$

Since $h \in C^{2}(\bar{\Omega})$ and $h \equiv 0$ in $\Omega \backslash \hat{\omega}$, we have

$$
\begin{gathered}
\left|I_{5}\right| \leq \epsilon \int_{t}^{t+T} \int_{0}^{s} g(s-\tau) \int_{\Gamma}|\Delta u(\tau)|^{2} d \Gamma d \tau d s+C(\epsilon) \int_{t}^{t+T} \int_{\hat{\omega} \cap \bar{\Omega}}|\Delta u|^{2} d x d s, \\
\left|\left(u^{\prime}(t+T), h \cdot \nabla u(t+T)\right)-\left(u^{\prime}(t), h \cdot \nabla u(t)\right)\right| \leq C(e(t)+e(t+T)), \\
\left|I_{6}\right|=\left|\int_{t}^{t+T} \int_{\Omega} \rho\left(x, u^{\prime}\right)(h \cdot \nabla u) d x d s\right| \leq C \int_{t}^{t+T} \int_{\Omega}\left|\rho\left(x, u^{\prime}\right)\right||\nabla u| d x d s
\end{gathered}
$$

and

$$
\begin{aligned}
\left|I_{7}\right| & =\left|\frac{1}{2} \int_{t}^{t+T} \int_{\Omega} \operatorname{divh}\left(\left|u^{\prime}\right|^{2}-|\Delta u|^{2}-M\left(|| \nabla u||^{2}\right)|\nabla u|^{2}\right) d x d s\right| \\
& \leq C \int_{t}^{t+T} \int_{\hat{\omega} \cap \bar{\Omega}}\left|u^{\prime}\right|^{2}+|\Delta u|^{2}+M\left(\left.|| \nabla u\right|^{2}\right)|\nabla u|^{2} d x d s
\end{aligned}
$$

Also using Hölder's and Poincare's inequalities, we obtain

$$
\begin{gathered}
\left|I_{8}\right|=\left|\int_{t}^{t+T} \int_{\Omega}(\Delta h \cdot \nabla u) \Delta u d x d s\right| \leq C \int_{t}^{t+T} \int_{\hat{\omega} \cap \bar{\Omega}}|\Delta u|^{2} d x d s \\
\left|I_{9}\right|=\left|2 \int_{t}^{t+T} \int_{\Omega} \Delta u \sum_{i, j=1}^{n} \frac{\partial h^{j}}{\partial x_{i}}\left(\frac{\partial}{\partial x_{i}} \frac{\partial u}{\partial x_{j}}\right) d x d s\right| \leq C \int_{t}^{t+T} \int_{\hat{\omega} \cap \bar{\Omega}}|\Delta u|^{2} d x d s
\end{gathered}
$$

and

$$
\begin{aligned}
& \left|I_{10}\right|=\left|\int_{t}^{t+T} M\left(\|\nabla u\|^{2}\right) \int_{\Omega} \sum_{i, j=1}^{n} \frac{\partial h^{j}}{\partial x_{i}} \frac{\partial u}{\partial x_{i}} \frac{\partial u}{\partial x_{j}} d x d s\right| \\
& \leq C \int_{t}^{t+T} \int_{\hat{\omega} \cap \bar{\Omega}} M\left(\|\nabla u\|^{2}\right)|\nabla u|^{2} d x d s .
\end{aligned}
$$


Similarly to $I_{1}$ and $I_{8}$ and by hypotheses of $g$, we have

$$
\begin{aligned}
& \int_{0}^{s} g(s-\tau)(\Delta u(\tau), \Delta h \cdot \nabla u(s)) d \tau \\
& \leq \frac{1}{2} \int_{\Omega}|\Delta h \cdot \nabla u|^{2} d x+\frac{1}{2}\|g\|_{L^{1}(0, \infty)}(g \square \Delta u)(s) \\
& +\int_{0}^{s} g(s-\tau) d \tau \int_{\Omega} \Delta u(s) \Delta h \cdot \nabla u(s) d x \\
& \leq C \int_{\hat{\omega} \cap \bar{\Omega}}|\Delta u|^{2} d x+\frac{1}{2}\|g\|_{L^{1}(0, \infty)}(g \square \Delta u)(s) .
\end{aligned}
$$

Hence,

$$
\begin{aligned}
\left|I_{11}\right| & =\left|\int_{t}^{t+T} \int_{0}^{s} g(s-\tau)(\Delta u(\tau), \Delta h \cdot \nabla u(s)) d \tau d s\right| \\
& \leq C \int_{t}^{t+T} \int_{\hat{\omega} \cap \bar{\Omega}}|\Delta u|^{2} d x d s+\frac{1}{2}\|g\|_{L^{1}(0, \infty)} \int_{t}^{t+T}(g \square \Delta u)(s) d s .
\end{aligned}
$$

By same method of $I_{11}$, we easily check that

$$
\begin{aligned}
\left|I_{12}\right| & =\left|\int_{t}^{t+T} \int_{0}^{s} g(s-\tau) \int_{\Omega} \Delta u(\tau) \sum_{i, j=1}^{n} \frac{\partial h^{j}}{\partial x_{i}}\left(\frac{\partial}{\partial x_{i}} \frac{\partial u(s)}{\partial x_{j}}\right) d x d \tau d s\right| \\
& \leq C \int_{t}^{t+T} \int_{\hat{\omega} \cap \bar{\Omega}}|\Delta u|^{2} d x d s+\frac{1}{2}\|g\|_{L^{1}(0, \infty)} \int_{t}^{t+T}(g \square \Delta u)(s) d s \\
\left|I_{13}\right| & =\left|\int_{t}^{t+T} \int_{0}^{s} g(s-\tau)(\Delta u(\tau), \nabla h \cdot \Delta u(s)) d \tau d s\right| \\
& \leq C \int_{t}^{t+T} \int_{\hat{\omega} \cap \bar{\Omega}}|\Delta u|^{2} d x d s+\frac{1}{2}\|g\|_{L^{1}(0, \infty)} \int_{t}^{t+T}(g \square \Delta u)(s) d s
\end{aligned}
$$

and

$$
\begin{aligned}
\left|I_{14}\right| & =\left|\int_{t}^{t+T} \int_{0}^{s} g(s-\tau)(\Delta u(\tau), h \cdot \nabla(\Delta u(s))) d \tau d s\right| \\
& \leq \epsilon \int_{t}^{t+T} \int_{\Omega}|\nabla(\Delta u)|^{2} d x d s+\frac{1}{2}\|g\|_{L^{1}(0, \infty)} C(\epsilon) \int_{t}^{t+T}(g \square \Delta u)(s) d s \\
& +C \int_{t}^{t+T} \int_{\hat{\omega} \cap \bar{\Omega}}|\Delta u|^{2} d x d s .
\end{aligned}
$$

Replacing (4.19) - (4.29) in (4.18) with $\epsilon>0$ sufficiently small and again calculating $I_{4}$, we obtain that

$$
\begin{aligned}
& \frac{1}{2} \int_{t}^{t+T} \int_{\Gamma_{1}}|\Delta u|^{2} d \Gamma d s \\
& \leq C\left\{e(t)+e(t+T)+\int_{t}^{t+T} \int_{\Omega}\left|\rho\left(x, u^{\prime}\right)\right||\nabla u| d x d s\right. \\
& \left.+\int_{t}^{t+T} \int_{\hat{\omega} \cap \bar{\Omega}}\left|u^{\prime}\right|^{2} d x d s+\int_{t}^{t+T} \int_{\hat{\omega} \cap \bar{\Omega}}|\Delta u|^{2}+M\left(\|\nabla u\|^{2}\right)|\nabla u|^{2} d x d s\right\} .
\end{aligned}
$$


In the sequel we will find boundedness for the last term of the right-hand side of (4.30). First, we use (4.7) with (4.6), then we can write that

$$
\begin{aligned}
& \int_{t}^{t+T} \int_{\Omega} \zeta\left(|\Delta u|^{2}+M\left(\left.|| \nabla u\right|^{2}\right)|\nabla u|^{2}\right) d x d s \\
& \leq \int_{t}^{t+T} \int_{\omega}\left|u^{\prime}\right|^{2} d x d s+\left(u^{\prime}(t), \zeta u(t)\right)-\left(u^{\prime}(t+T), \zeta u(t+T)\right) \\
& -\int_{t}^{t+T} \int_{\Omega} \rho\left(x, u^{\prime}\right) \zeta u d x d s \\
& \quad-\int_{t}^{t+T} M\left(\| \nabla u||^{2}\right) \int_{\Omega} u(\nabla u \cdot \nabla \zeta) d x d s \\
& -\int_{t}^{t+T} \int_{\omega}(\Delta u \Delta \zeta u+2 \Delta u(\nabla \zeta \cdot \nabla u)) d x d s \\
& \quad+\int_{t}^{t+T} \int_{0}^{s} g(s-\tau)(\Delta u(\tau), \Delta \zeta u(s)+2 \nabla \zeta \cdot \nabla u(s) \\
& +\zeta \Delta u(s)) d \tau d s \\
& :=\int_{t}^{t+T} \int_{\omega}\left|u^{\prime}\right|^{2} d x d s+\left(u^{\prime}(t), \zeta u(t)\right) \\
& -\left(u^{\prime}(t+T), \zeta u(t+T)\right)-I_{15}-I_{16}-I_{17}+I_{18} .
\end{aligned}
$$

Similarly to (4.20), we get

$$
\left|\left(u^{\prime}(t), \zeta u(t)\right)-\left(u^{\prime}(t+T), \zeta u(t+T)\right)\right| \leq C(e(t)+e(t+T)) .
$$

Assumption on $\zeta$ (see (4.6)) and using Höler's and Poincare's inequalities, we obtain

$$
\left|I_{15}\right|=\left|\int_{t}^{t+T} \int_{\Omega} \rho\left(x, u^{\prime}\right) \zeta u d x d s\right| \leq \int_{t}^{t+T} \int_{\Omega}\left|\rho\left(x, u^{\prime}\right)\right||u| d x d s
$$

$$
\begin{aligned}
\left|I_{16}\right| & =\left|\int_{t}^{t+T} M\left(\|\left.\nabla u\right|^{2}\right) \int_{\Omega} u(\nabla u \cdot \nabla \zeta) d x d s\right| \\
& \leq \int_{t}^{t+T} M\left(\left.|| \nabla u\right|^{2}\right)\left[\int_{\omega} \zeta^{*}|u|^{2} d x+\int_{\omega} \frac{1}{4 \zeta^{*}}|\nabla u|^{2}|\nabla \zeta|^{2} d x\right] d s \\
& \leq C \int_{t}^{t+T} \int_{\omega}|u|^{2} d x d s+\frac{1}{4} \int_{t}^{t+T} \int_{\Omega} M\left(\left.|| \nabla u\right|^{2}\right) \zeta|\nabla u|^{2} d x d s
\end{aligned}
$$


and

$$
\begin{aligned}
\left|I_{17}\right| & =\left|\int_{t}^{t+T} \int_{\omega}(\Delta u \Delta \zeta u+2 \Delta u(\nabla \zeta \cdot \nabla u)) d x d s\right| \\
& \leq \int_{t}^{t+T} \int_{\omega} \sqrt{\frac{2}{\zeta}}|\Delta \zeta||u| \sqrt{\frac{\zeta}{2}}|\Delta u| \\
& +2 \frac{|\nabla \zeta|}{\sqrt{\zeta}}|\nabla u| \sqrt{\zeta}|\Delta u| d x d s \\
& \leq \zeta^{*} \int_{t}^{t+T} \int_{\omega}|u|^{2} d x d s+2 \zeta^{*} \int_{t}^{t+T} \int_{\omega}|\nabla u|^{2} d x d s \\
& +\frac{3}{4} \int_{t}^{t+T} \int_{\Omega} \zeta|\Delta u|^{2} d x d s
\end{aligned}
$$

where $\zeta^{*}=\max \left\{\sup _{x \in \Omega} \frac{|\nabla \zeta(x)|^{2}}{\zeta(x)}, \sup _{x \in \Omega} \frac{|\Delta \zeta(x)|^{2}}{\zeta(x)}\right\}$.

Similarly to $I_{1}$ and $I_{17}$ and using $a b \leq a^{2}+\frac{1}{4} b^{2}$ we have

$$
\begin{aligned}
\left|I_{18}\right|= & \left|\int_{t}^{t+T} \int_{0}^{s} g(s-\tau)(\Delta u(\tau), \Delta \zeta u(s)+2 \nabla \zeta \cdot \nabla u(s)+\zeta \Delta u(s)) d \tau d s\right| \\
\leq & \left(2 \zeta^{*}+1\right) \int_{t}^{t+T}(g \square \Delta u)(s) d s+\left(\zeta^{*}+\frac{1}{4}\right) \int_{t}^{t+T} \int_{\omega}|u|^{2} d x d s \\
& +\left(2 \zeta^{*}+1\right) \int_{t}^{t+T} \int_{\omega}|\nabla u|^{2} d x d s+\int_{t}^{t+T} \int_{\Omega} \zeta|\Delta u|^{2} d x d s .
\end{aligned}
$$

Replacing (4.32) - (4.36) in (4.31) and again calculating $I_{4}$, we obtain that

$$
\begin{aligned}
& \int_{t}^{t+T} \int_{\Omega} \zeta\left(|\Delta u|^{2}+M\left(|| \nabla u||^{2}\right)|\nabla u|^{2}\right) d x d s \\
& \leq C\left\{e(t)+e(t+T)+\int_{t}^{t+T} \int_{\Omega}\left|\rho\left(x, u^{\prime}\right)\right||u| d x d s\right. \\
& \left.+\int_{t}^{t+T} \int_{\omega}\left|u^{\prime}\right|^{2}+|u|^{2}+|\nabla u|^{2} d x d s\right\} .
\end{aligned}
$$

Moreover, since $0 \leq \zeta(x) \leq 1$, it follows that

$$
\begin{aligned}
& \int_{t}^{t+T} \int_{\hat{\omega} \cap \bar{\Omega}}|\Delta u|^{2}+M\left(|| \nabla u||^{2}\right)|\nabla u|^{2} d x d s \\
& \leq C\left\{e(t)+e(t+T)+\int_{t}^{t+T} \int_{\Omega}\left|\rho\left(x, u^{\prime}\right)\right||u| d x d s\right. \\
& \left.+\int_{t}^{t+T} \int_{\omega}\left|u^{\prime}\right|^{2}+|u|^{2}+|\nabla u|^{2} d x d s\right\} .
\end{aligned}
$$


Finally, noting that $\hat{\omega} \cap \bar{\Omega} \subset \omega$, replacing (4.37) in (4.30) we get

$$
\begin{aligned}
& \frac{1}{2} \int_{t}^{t+T} \int_{\Gamma_{1}}|\Delta u|^{2} d \Gamma d s \\
& \leq C\left\{e(t)+e(t+T)+\int_{t}^{t+T} \int_{\Omega}\left|\rho\left(x, u^{\prime}\right)\right|(|u|+|\nabla u|) d x d s\right. \\
& \left.\quad+\int_{t}^{t+T} \int_{\omega}\left|u^{\prime}\right|^{2}+|u|^{2}+|\nabla u|^{2} d x d s\right\} .
\end{aligned}
$$

Thus we replace (4.38) in (4.17) and take $T \geq \frac{2 C}{\gamma}+1$, then the proof of Proposition 4.1 is completed.

Proposition 4.2. Let $u$ be the solution of (1.1) and $\Delta e$ be given by

$$
\Delta e \equiv e(t)-e(t+T)
$$

Then, for $T>0$ given in Proposition 4.1, the followings hold:

case 1

If $r \geq 0,0 \leq p \leq \frac{2}{n-2}$ and $n \geq 3$, then

$$
\int_{t}^{t+T} \int_{\Omega}\left|\rho\left(x, u^{\prime}\right)\right|(|u|+|\nabla u|) d x d s \leq C(\Delta e)^{\frac{1}{r+2}} \sqrt{e(t)}+C(\Delta e)^{\frac{p+1}{p+2}} \sqrt{e(t)} .
$$

When $n=2$, this estimate holds for the case $r \geq 0$ and $p \geq 0$.

case 2

If $r \geq 0,-1<p<0$ and $n \geq 2$, then

$\int_{t}^{t+T} \int_{\Omega}\left|\rho\left(x, u^{\prime}\right)\right|(|u|+|\nabla u|) d x d s \leq C(\Delta e)^{\frac{1}{r+2}} \sqrt{e(t)}+C(\Delta e)^{\frac{1}{2(p+2)}} \sqrt{e(t)}$.

case 3

If $-1<r<0,0 \leq p \leq \frac{2}{n-2}$ and $n \geq 3$, then

$$
\int_{t}^{t+T} \int_{\Omega}\left|\rho\left(x, u^{\prime}\right)\right|(|u|+|\nabla u|) d x d s \leq C(\Delta e)^{\frac{r+1}{r+2}} \sqrt{e(t)}+C(\Delta e)^{\frac{p+1}{p+2}} \sqrt{e(t)} .
$$

When $n=2$, this estimate holds for the case $-1<r<0$ and $p \geq 0$.

case 4

If $-1<r<0,-1<p<0$ and $n \geq 2$, then

$\int_{t}^{t+T} \int_{\Omega}\left|\rho\left(x, u^{\prime}\right)\right|(|u|+|\nabla u|) d x d s \leq C(\Delta e)^{\frac{r+1}{r+2}} \sqrt{e(t)}+C(\Delta e)^{\frac{1}{2(p+2)}} \sqrt{e(t)}$.

For $n=1$ the above estimates are the same as for the case $n=2$.

Proof. By the hypotheses on $\rho$, we have

$$
\begin{aligned}
& \int_{t}^{t+T} \int_{\Omega}\left|\rho\left(x, u^{\prime}\right)\right|(|u|+|\nabla u|) d x d s \\
& \leq \int_{t}^{t+T} \int_{\Omega_{1}} c_{5} a(x)\left(\left|u^{\prime}\right|^{r+1}+\left|u^{\prime}\right|\right)(|u|+|\nabla u|) d x d s \\
& +\int_{t}^{t+T} \int_{\Omega_{2}} c_{7} a(x)\left(\left|u^{\prime}\right|^{p+1}+\left|u^{\prime}\right|\right)(|u|+|\nabla u|) d x d s \\
& :=I_{19}+I_{20},
\end{aligned}
$$

where $\Omega_{1}=\left\{(x, t) \in \Omega \times \mathbb{R}_{+}:\left|u^{\prime}\right| \leq 1\right\}$ and $\Omega_{2}=\Omega \backslash \Omega_{1}$. 
Now, we will estimate $I_{19}$ and $I_{20}$.

(i) Estimating $I_{19}$ for $r \geq 0$ and $n \geq 2$.

In this case we see, by Poincare's inequality, Sobolev imbedding theorem,(2.6) and (4.3),

$$
\begin{aligned}
I_{19} & \leq 2 c_{5}\|\sqrt{a(x)}\|_{L^{\infty}(\Omega)} \int_{t}^{t+T} \int_{\Omega_{1}} \sqrt{a(x)}\left|u^{\prime}\right|(|u|+|\nabla u|) d x d s \\
& \leq C\left(\int_{t}^{t+T} \int_{\Omega_{1}} a(x)\left|u^{\prime}\right|^{2} d x d s\right)^{\frac{1}{2}}\left(\int_{t}^{t+T} e(s) d s\right)^{\frac{1}{2}} \\
& \leq C\left(\int_{t}^{t+T} \int_{\Omega_{1}} a(x)\left|u^{\prime}\right|^{r+2} d x d s\right)^{\frac{1}{r+2}} \sqrt{e(t)} \\
& \leq C\left(\int_{t}^{t+T} \int_{\Omega_{1}} \rho\left(x, u^{\prime}\right) u^{\prime} d x d s\right)^{\frac{1}{r+2}} \sqrt{e(t)} \\
& \leq C(\Delta e)^{\frac{1}{r+2}} \sqrt{e(t)} .
\end{aligned}
$$

(ii) Estimating $I_{19}$ for $-1 \leq r \leq 0$ and $n \geq 2$.

Similarly to $(i)$ and using $L^{2} \hookrightarrow L^{r+2}$, we have

$$
\begin{aligned}
I_{19} & \leq 2 c_{5} \int_{t}^{t+T} \int_{\Omega_{1}} a(x)\left|u^{\prime}\right|^{r+1}(|u|+|\nabla u|) d x d s \\
& \leq C\left(\int_{t}^{t+T} \int_{\Omega_{1}} a(x)\left|u^{\prime}\right|^{r+2} d x d s\right)^{\frac{r+1}{r+2}} \\
& \left(\int_{t}^{t+T} \int_{\Omega_{1}}(|u|+|\nabla u|)^{r+2} d x d s\right)^{\frac{1}{r+2}} \\
& \leq C\left(\int_{t}^{t+T} \int_{\Omega_{1}} \rho\left(x, u^{\prime}\right) u^{\prime} d x d s\right)^{\frac{1}{r+2}} \\
& \left(\int_{t}^{t+T} \int_{\Omega_{1}}(|u|+|\nabla u|)^{2} d x d s\right)^{\frac{1}{2}} \\
& \leq C(\Delta e)^{\frac{r+1}{r+2}} \sqrt{e(t) .}
\end{aligned}
$$

(iii) Estimating $I_{20}$ for $0 \leq p \leq \frac{2}{n-2}$ and $n \geq 3$. 
By Hölder's inequality and Sobolev imbedding theorem, we get

$$
\begin{aligned}
I_{20} & \leq 2 c_{7} \int_{t}^{t+T} \int_{\Omega_{2}} a(x)\left|u^{\prime}\right|^{p+1}(|u|+|\nabla u|) d x d s \\
& \leq C\left(\int_{t}^{t+T} \int_{\Omega_{2}} a(x)\left|u^{\prime}\right|^{p+2} d x d s\right)^{\frac{p+1}{p+2}} \\
& \left(\int_{t}^{t+T} \int_{\Omega_{2}}(|u|+|\nabla u|)^{p+2} d x d s\right)^{\frac{1}{p+2}} \\
& \leq C\left(\int_{t}^{t+T} \int_{\Omega_{2}} a(x)\left|u^{\prime}\right|^{p+2} d x d s\right)^{\frac{p+1}{p+2}} \\
& \left(\int_{t}^{t+T} \int_{\Omega}|\nabla u|^{p+2} d x d s\right)^{\frac{1}{p+2}} .
\end{aligned}
$$

Now we use the Lemma 2.1, then

$$
\begin{aligned}
\|\nabla u\|_{L^{p+2}(\Omega)} & \leq C\|\nabla u\|_{H^{1}(\Omega)}^{\theta}\|\nabla u\|_{L^{2}(\Omega)}^{1-\theta} \\
& \leq C\|\Delta u\|_{L^{2}(\Omega)}^{\theta}\|\nabla u\|_{L^{2}(\Omega)}^{1-\theta} \leq C\left(\frac{2}{\ell} e(t)\right)^{\frac{\theta}{2}}\left(\frac{2}{s_{0}} e(t)\right)^{\frac{1-\theta}{2}} \leq C \sqrt{e(t)} .
\end{aligned}
$$

Replacing above inequality in (4.42), it follows that

$$
I_{20} \leq C\left(\int_{t}^{t+T} \int_{\Omega_{2}} \rho\left(x, u^{\prime}\right) u^{\prime} d x d s\right)^{\frac{p+1}{p+2}} \sqrt{e(t)} \leq C(\Delta e)^{\frac{p+1}{p+2}} \sqrt{e(t)}
$$

If $n=2$, then we can obtain same result for $p \geq 0$.

(iv) Estimating $I_{20}$ for $-1<p<0$ and $n \geq 2$.

By Hölder's and Poincare's inequalities, we have

$$
\begin{aligned}
I_{20} & \leq 2 c_{7} \int_{t}^{t+T} \int_{\Omega_{2}} a(x)\left|u^{\prime}\right|(|u|+|\nabla u|) d x d s \\
& \leq C\left(\int_{t}^{t+T} \int_{\Omega_{2}} a(x)\left|u^{\prime}\right|^{2} d x d s\right)^{\frac{1}{2}}\left(\int_{t}^{t+T} \int_{\Omega_{2}}|\nabla u|^{2} d x d s\right)^{\frac{1}{2}} \\
& \leq C\left(\int_{t}^{t+T} \int_{\Omega_{2}}\left|u^{\prime}\right|^{\frac{p+2}{p+1}} d x d s\right)^{\frac{p+1}{p+2}} \\
& \left(\int_{t}^{t+T} \int_{\Omega_{2}} a(x)\left|u^{\prime}\right|^{p+2} d x d s\right)^{\frac{1}{2(p+2)}}\left(\int_{t}^{t+T} e(s) d s\right)^{\frac{1}{2}} \\
& \leq C\left(\int_{t}^{t+T} \int_{\Omega_{2}} \rho\left(x, u^{\prime}\right) u^{\prime} d x d s\right)^{\frac{1}{2(p+2)}} \sqrt{e(t)} \\
& \leq C(\Delta e)^{\frac{1}{2(p+2)} \sqrt{e(t)}}
\end{aligned}
$$

because $u^{\prime} \in L^{\infty}\left(0, \infty ; H^{1}(\Omega)\right) \hookrightarrow L^{\infty}\left(0, \infty ; L^{\frac{p+2}{p+1}}(\Omega)\right)$.

Therefore, replacing (4.40), (4.41), (4.43) and (4.44) in (4.39) we conclude the proof of Proposition 4.2.

Using Young's inequality and Propositions 4.1, 4.2, we obtain the next result. 
Proposition 4.3. Let $u$ be the solution of (1.1). Then for $T>0$ given in Proposition 4.1, the modified energy associated with (1.1) satisfies

$$
e(t) \leq C\left\{A_{i}(t)^{2}+\int_{t}^{t+T} \int_{\omega}\left(\left|u^{\prime}\right|^{2}+|u|^{2}+|\nabla u|^{2}\right) d x d s\right\}
$$

,for $i=1,2,3,4$, where

case 1

If $r \geq 0$ and $0 \leq p \leq \frac{2}{n-2} \quad(0 \leq p<\infty$ if $n=2)$,

$$
A_{1}(t)^{2}=\Delta e+(\Delta e)^{\frac{2}{r+2}}+(\Delta e)^{\frac{2(p+1)}{p+2}} .
$$

case 2

If $r \geq 0$ and $-1<p<0$,

$$
A_{2}(t)^{2}=\Delta e+(\Delta e)^{\frac{2}{r+2}}+(\Delta e)^{\frac{1}{p+2}} .
$$

case 3

If $-1<r<0$ and $0 \leq p \leq \frac{2}{n-2}(0 \leq p<\infty$ if $n=2)$,

$$
A_{3}(t)^{2}=\Delta e+(\Delta e)^{\frac{2(r+1)}{r+2}}+(\Delta e)^{\frac{2(p+1)}{p+2}}
$$

case 4

If $-1<r<0$ and $-1<p<0$,

$$
A_{4}(t)^{2}=\Delta e+(\Delta e)^{\frac{2(r+1)}{r+2}}+(\Delta e)^{\frac{1}{p+2}} .
$$

To arrive at the desired difference inequality on $e(t)$ we must estimate further the last two terms in (4.45). Concerning the last two terms of the right hand side in (4.45) we show :

Proposition 4.4. According to each $A_{i}(t)^{2}$ given in Proposition 4.3 there exists a constant $C>0$ such that

$$
\int_{t}^{t+T} \int_{\Omega}\left(|u|^{2}+|\nabla u|^{2}\right) d x d s \leq C\left\{A_{i}(t)^{2}+\int_{t}^{t+T} \int_{\omega}\left|u^{\prime}\right|^{2} d x d s\right\} .
$$

Before the proof of Proposition 4.4, we shall show the following result.

Lemma 4.1. Consider $\varphi \in W^{1, \infty}(0, T), \varphi \geq 0$. Then if the function

$$
v \in W^{1, \infty}\left(0, T ; L^{2}(\Omega)\right) \cap L^{\infty}(0, T ; V)
$$

satisfies the conditions

$$
\left\{\begin{array}{l}
v_{t t}+\Delta^{2} v-\varphi(t) \Delta v-\int_{0}^{t} g(t-\tau) \Delta^{2} v(\tau) d \tau=0 \quad \text { in } \quad \Omega \times(0, T), \\
v=\frac{\partial v}{\partial \nu}=0 \quad \text { on } \quad \Gamma \times(0, T), \\
v_{t}=0 \quad \text { in } \quad \omega \times(0, T),
\end{array}\right.
$$

we have that $v \equiv 0$ in $\Omega \times(0, T)$.

Proof. If $\varphi(t)=\varphi_{0}$ (constant), for any $t \in[0, T]$, by taking the derivative of (4.47) with respect to $t$ we obtain that $w=v_{t}$ satisfies (in the distributions sense) the equation

$$
\left\{\begin{array}{l}
w_{t t}+\Delta^{2} w-\varphi_{0} \Delta w-g(0) \Delta^{2} v-\int_{0}^{t} g^{\prime}(t-\tau) \Delta^{2} v(\tau) d \tau=0 \quad \text { in } \quad \Omega \times(0, T), \\
w=\frac{\partial w}{\partial \nu}=0 \quad \text { on } \quad \Gamma \times(0, T), \\
w=0 \quad \text { in } \quad \omega \times(0, T) .
\end{array}\right.
$$


By $\left(H_{5}\right)$ we have that $v_{t}=w \equiv 0$ in $\Omega \times(0, T)$. From (4.47) it follows that

$$
\left\{\begin{array}{l}
\Delta^{2} v-\varphi_{0} \Delta v-\int_{0}^{t} g(t-\tau) \Delta^{2} v(\tau) d \tau=0 \quad \text { in } \quad \Omega \times(0, T), \\
v=\frac{\partial v}{\partial \nu}=0 \quad \text { on } \quad \Gamma \times(0, T) .
\end{array}\right.
$$

By the fact $v_{t}=0,(2.3)$ and standard elliptic uniqueness result, it follows that the above equation imply the conclusion of the Lemma.

Now let us suppose that $\varphi_{t}(t) \neq 0$ for $t$ varying in a subset of strictly positive measure of $[0, T]$. By (4.47) and the fact that $v(x, t)=v(x)$ if $x \in \omega$ we get

$$
\left\{\begin{array}{l}
\left(1-\int_{0}^{t} g(t-\tau) d \tau\right) \Delta^{2} v-\varphi(t) \Delta v=0 \quad \text { in } \quad \omega \times(0, T), \\
v=\frac{\partial v}{\partial \nu}=0 \quad \text { on } \quad \Gamma \times(0, T) .
\end{array}\right.
$$

Applying to (2.3), and then deriving above equation with respect to $t$, we have

$$
\Delta v=0 \quad \text { in } \quad \omega
$$

since $\varphi_{t}(t) \neq 0$. Hence by Holmgren's uniqueness theorem, we obtain that

$$
v \equiv 0 \quad \text { in } \quad \omega .
$$

We can use $\left(H_{5}\right)$ again with $\alpha=0$ to obtain that

$$
v \equiv 0 \quad \text { in } \quad \Omega \text {. }
$$

Proof of Proposition 4.4. We prove (4.46) by contradiction. If (4.46) was false, there exist a sequence $\left\{t_{n}\right\} \subset \mathbb{R}$ and let $\left\{u_{n}(0), u_{n}^{\prime}(0)\right\}$ be a sequence of initial data where the corresponding solutions $\left\{u_{n}\right\}$ of (1.1) with $E_{n}(0)$ uniform bounded in $n$, verifies

$$
\lim _{n \rightarrow \infty} \frac{\int_{t_{n}}^{t_{n}+T} \int_{\Omega}\left(\left|u_{n}\right|^{2}+\left|\nabla u_{n}\right|^{2}\right) d x d s}{A_{i}\left(t_{n}\right)^{2}+\int_{t_{n}}^{t_{n}+T} \int_{\omega}\left|u_{n}^{\prime}\right|^{2} d x d s}=\infty
$$

Setting

$$
\lambda_{n}^{2}=\int_{t_{n}}^{t_{n}+T} \int_{\Omega}\left(\left|u_{n}\right|^{2}+\left|\nabla u_{n}\right|^{2}\right) d x d s
$$

and

$$
v_{n}(t)=\frac{u_{n}\left(t+t_{n}\right)}{\lambda_{n}}, 0 \leq t \leq T
$$

Then, we get

$$
Q_{n}^{2}:=\frac{1}{\lambda_{n}^{2}}\left\{A_{i}\left(t_{n}\right)^{2}+\int_{t_{n}}^{t_{n}+T} \int_{\omega}\left|u_{n}^{\prime}\right|^{2} d x d s\right\} \rightarrow 0 \quad \text { as } \quad n \rightarrow \infty
$$

and

$$
\int_{0}^{T} \int_{\Omega}\left(\left|v_{n}\right|^{2}+\left|\nabla v_{n}\right|^{2}\right) d x d s=1
$$


Thus, we have from (4.49), (4.50) and Proposition 4.3,

$$
\begin{aligned}
e\left(v_{n}(t)\right) & =e\left(\frac{u_{n}\left(t+t_{n}\right)}{\lambda_{n}^{2}}\right)=\frac{1}{\lambda_{n}^{2}} e\left(u_{n}\left(t+t_{n}\right)\right) \leq \frac{1}{\lambda_{n}^{2}} e\left(u_{n}\left(t_{n}\right)\right) \\
& \leq \frac{C}{\lambda_{n}^{2}}\left\{A_{i}\left(t_{n}\right)^{2}+\int_{t_{n}}^{t_{n}+T} \int_{\omega}\left|u_{n}^{\prime}\right|^{2} d x d s+\int_{t_{n}}^{t_{n}+T} \int_{\Omega}\left(\left|u_{n}\right|^{2}+\left|\nabla u_{n}\right|^{2}\right) d x d s\right\} \\
& =C\left\{Q_{n}^{2}+\int_{0}^{T} \int_{\Omega}\left(\left|v_{n}\right|^{2}+\left|\nabla v_{n}\right|^{2}\right) d x d s\right\} \\
& \leq C .
\end{aligned}
$$

Therefore,

$$
\left\|v_{n}^{\prime}\right\|,\left\|\nabla v_{n}\right\|,\left\|\Delta v_{n}\right\| \leq C .
$$

Furthermore, using Poincare's inequality we obtain

$$
\begin{aligned}
& \int_{\Omega}\left|v_{n}(x, t)\right|^{2} d x=\int_{\Omega} \frac{1}{\lambda_{n}^{2}}\left|u_{n}\left(x, t+t_{n}\right)\right|^{2} d x \\
& \leq C \int_{\Omega} \frac{1}{\lambda_{n}^{2}}\left|\nabla u_{n}\left(x, t+t_{n}\right)\right|^{2} d x=C \int_{\Omega}|\nabla v(x, t)|^{2} d x \leq C .
\end{aligned}
$$

Combining the above estimates, we deduce that

$$
\left\{v_{n}\right\} \quad \text { is bounded in } \quad W^{1, \infty}\left(0, T ; L^{2}(\Omega)\right) \cap L^{\infty}(0, T ; V) .
$$

In order to take the limit of $\left\{v_{n}\right\}$ we shall first check that

$$
\lim _{n \rightarrow \infty} \frac{1}{\lambda_{n}} \rho\left(x, u^{\prime}\left(t+t_{n}\right)\right)=0 \quad \text { in } \quad L^{1}([0, T] \times \Omega) .
$$

Indeed, we consider in four cases (This divided cases is the same cases as Proposition $4.3)$.

For the case $r \geq 0$ and $0 \leq p \leq \frac{2}{n-2}$ if $n>2$ and $0 \leq p<\infty$ if $n=1,2$, by Proposition 4.2 (see case 1 ) and the definition of $A_{1}(t)$, we easily check that

$$
\int_{t}^{t+T} \int_{\Omega}\left|\rho\left(x, u^{\prime}\right)\right| d x d s \leq C\left((\Delta e)^{\frac{1}{r+2}}+(\Delta e)^{\frac{p+1}{p+2}}\right) \leq C\left(A_{1}(t)+A_{1}(t)\right)=2 C A_{1}(t) .
$$

Hence,

$$
\frac{1}{\lambda_{n}} \int_{t_{n}}^{t_{n}+T} \int_{\Omega}\left|\rho\left(x, u_{n}^{\prime}\right)\right| d x d s \leq C \leq C Q_{n} \rightarrow 0 \quad \text { as } \quad n \rightarrow \infty .
$$

The remaining cases are treated similarly. We have proved that

$$
\frac{1}{\lambda_{n}} \rho\left(x, u^{\prime}\left(t+t_{n}\right)\right) \rightarrow 0 \quad \text { in } \quad L^{1}([0, T] \times \Omega) .
$$

Therefore, (4.52) is proved.

Now, using (4.51) and the Aubin-Lion's Lemma, there exists a function $v$ and a subsequence, still denoted by $\left\{v_{n}\right\}$, such that

$$
v_{n} \rightarrow v \quad \text { in } \quad L^{2}\left(0, T ; H^{1}(\Omega)\right),
$$

and by (4.50), we have

$$
\|v\|_{L^{2}\left(0, T ; H^{1}(\Omega)\right)}=1
$$


Furthermore, from (4.49) we get

$$
\int_{0}^{T} \int_{\omega}\left|v^{\prime}\right|^{2} d x d s=0
$$

Then according to the previous analysis, the limit function $v$ satisfies (4.54)

$$
\left\{\begin{array}{l}
v \in W^{1, \infty}\left(0, T ; L^{2}(\Omega)\right) \cap L^{\infty}(0, T ; V), \\
v_{t t}+\Delta^{2} v-M\left(\|\nabla u\|^{2}\right) \Delta v-\int_{0}^{t} g(t-\tau) \Delta^{2} v(\tau) d \tau=0 \quad \text { in } \quad \Omega \times(0, T), \\
v=\frac{\partial v}{\partial \nu}=0 \quad \text { on } \quad \Gamma \times(0, T) \\
v_{t}=0 \quad \text { in } \quad \omega \times(0, T)
\end{array}\right.
$$

So, by Lemma 4.1 we have $v \equiv 0$ in $\Omega \times(0, T)$. This is a contradiction to (4.53). We complete the proof of Proposition 4.4.

4.2. Proof of the Theorem 2.2. Combining Proposition 4.3 and 4.4 , we have

$$
e(t) \leq C\left\{A_{i}(t)^{2}+\int_{t}^{t+T} \int_{\omega}\left|u^{\prime}\right|^{2} d x d s\right\}
$$

where $A_{i}(t), i=1,2,3,4$, are given in Proposition 4.3.

Finally, we shall estimate the last term in (4.55) and derive the decay estimates stated in Theorem.

case $1: r \geq 0,0 \leq p \leq \frac{2}{n-2}$ and $n>2(0 \leq p<\infty$ if $n=1,2)$.

By hypothesis on $a(x)$, it follows that (see (4.40))

$$
\begin{aligned}
& \int_{t}^{t+T} \int_{\omega}\left|u^{\prime}\right|^{2} d x d s \\
& \leq \frac{1}{a_{0}} \int_{t}^{t+T} \int_{\Omega} a(x)\left|u^{\prime}\right|^{2} d x d s \\
& \leq C\left\{\int_{t}^{t+T} \int_{\Omega_{1}} a(x)\left|u^{\prime}\right|^{2} d x d s+\int_{t}^{t+T} \int_{\Omega_{2}} a(x)\left|u^{\prime}\right|^{2} d x d s\right\} \\
& \leq C\left\{\left(\int_{t}^{t+T} \int_{\Omega_{1}} a(x)\left|u^{\prime}\right|^{r+2} d x d s\right)^{\frac{2}{r+2}}+\int_{t}^{t+T} \int_{\Omega_{2}} a(x)\left|u^{\prime}\right|^{p+2} d x d s\right\} \\
& \leq C\left\{\left(\int_{t}^{t+T} \int_{\Omega_{1}} \rho\left(x, u^{\prime}\right) u^{\prime} d x d s\right)^{\frac{2}{r+2}}+\int_{t}^{t+T} \int_{\Omega_{2}} \rho\left(x, u^{\prime}\right) u^{\prime} d x d s\right\} \\
& \leq C\left((\Delta e)^{\frac{2}{r+2}}+\Delta e\right) .
\end{aligned}
$$

Hence, we have from (4.55) and the definition of $A_{1}(t)^{2}$

$$
e(t) \leq C\left\{\Delta e+(\Delta e)^{\frac{2}{r+2}}+(\Delta e)^{\frac{2(p+1)}{p+2}}\right\} \leq C(\Delta e)^{\kappa_{1}}
$$

where $\kappa_{1}=\min \left\{\frac{2}{r+2}, \frac{2(p+1)}{p+2}\right\}=\frac{2}{r+2}$. Therefore,

$$
\sup _{t \leq s \leq t+T} e(s)^{\frac{1}{\kappa_{1}}} \leq C(e(t)-e(t+T)) .
$$


Applying Lemma 2.2 to (4.56) we conclude

$$
e(t) \leq C(1+t)^{-\eta_{1}}
$$

with $\eta_{1}=\frac{2}{r}$.

case $2: r \geq 0$ and $-1<p<0$.

$$
\begin{aligned}
& \int_{t}^{t+T} \int_{\omega}\left|u^{\prime}\right|^{2} d x d s \\
& \leq C\left\{\int_{t}^{t+T} \int_{\Omega_{1}} a(x)\left|u^{\prime}\right|^{2} d x d s+\int_{t}^{t+T} \int_{\Omega_{2}} a(x)\left|u^{\prime}\right|^{2} d x d s\right\} \\
& \leq C\left\{\left(\int_{t}^{t+T} \int_{\Omega_{1}} a(x)\left|u^{\prime}\right|^{r+2} d x d s\right)^{\frac{2}{r+2}}+\left(\int_{t}^{t+T} \int_{\Omega_{2}} a(x)\left|u^{\prime}\right|^{p+2} d x d s\right)^{\frac{1}{p+2}}\right\} \\
& \leq C\left((\Delta e)^{\frac{2}{r+2}}+(\Delta e)^{\frac{1}{p+2}}\right) .
\end{aligned}
$$

Hence, we have from (4.55) and the definition of $A_{2}(t)^{2}$

$$
e(t) \leq C\left\{\Delta e+(\Delta e)^{\frac{2}{r+2}}+(\Delta e)^{\frac{1}{p+2}}\right\} \leq C(\Delta e)^{\kappa_{2}},
$$

where $\kappa_{2}=\min \left\{\frac{2}{r+2}, \frac{1}{p+2}\right\}$. Therefore,

$$
\sup _{t \leq s \leq t+T} e(s)^{\frac{1}{\kappa_{2}}} \leq C(e(t)-e(t+T)) .
$$

Applying Lemma 2.2 to (4.58) we conclude

$$
e(t) \leq C(1+t)^{-\eta_{2}}
$$

with $\eta_{2}=\min \left\{\frac{2}{r}, \frac{1}{p+1}\right\}$.

case 3 : $-1<r<0,0 \leq p \leq \frac{2}{n-2}$ and $n>2(0 \leq p<\infty$ if $n=2)$.

$$
\begin{aligned}
& \int_{t}^{t+T} \int_{\omega}\left|u^{\prime}\right|^{2} d x d s \\
& \leq C\left\{\int_{t}^{t+T} \int_{\Omega_{1}} a(x)\left|u^{\prime}\right|^{2} d x d s+\int_{t}^{t+T} \int_{\Omega_{2}} a(x)\left|u^{\prime}\right|^{2} d x d s\right\} \\
& \leq C\left\{\left(\int_{t}^{t+T} \int_{\Omega_{1}} a(x)\left|u^{\prime}\right|^{r+2} d x d s\right)^{\frac{1}{r+2}}+\int_{t}^{t+T} \int_{\Omega_{2}} a(x)\left|u^{\prime}\right|^{p+2} d x d s\right\} \\
& \leq C\left((\Delta e)^{\frac{1}{r+2}}+(\Delta e)\right) .
\end{aligned}
$$

Hence, we have from (4.55) and the definition of $A_{3}(t)^{2}$

$$
e(t) \leq C\left\{\Delta e+(\Delta e)^{\frac{1}{r+2}}+(\Delta e)^{\frac{2(r+1)}{r+2}}+(\Delta e)^{\frac{2(p+1)}{p+2}}\right\} \leq C(\Delta e)^{\kappa_{3}},
$$

where $\kappa_{3}=\min \left\{\frac{1}{r+2}, \frac{2(r+1)}{r+2}\right\}$. Therefore,

$$
\sup _{t \leq s \leq t+T} e(s)^{\frac{1}{\kappa_{3}}} \leq C(e(t)-e(t+T)) .
$$


Applying Lemma 2.2 to (4.60) we conclude

$$
e(t) \leq C(1+t)^{-\eta_{3}}
$$

with $\eta_{3}=\min \left\{\frac{1}{r+1}, \frac{-r}{2(r+1)}\right\}$.

case $4:-1<r<0$ and $-1<p<0$.

$$
\begin{aligned}
& \int_{t}^{t+T} \int_{\omega}\left|u^{\prime}\right|^{2} d x d s \\
& \leq C\left\{\int_{t}^{t+T} \int_{\Omega_{1}} a(x)\left|u^{\prime}\right|^{2} d x d s+\int_{t}^{t+T} \int_{\Omega_{2}} a(x)\left|u^{\prime}\right|^{2} d x d s\right\} \\
& \leq C\left\{\left(\int_{t}^{t+T} \int_{\Omega_{1}} a(x)\left|u^{\prime}\right|^{r+2} d x d s\right)^{\frac{1}{r+2}}+\left(\int_{t}^{t+T} \int_{\Omega_{2}} a(x)\left|u^{\prime}\right|^{p+2} d x d s\right)^{\frac{1}{p+2}}\right\} \\
& \leq C\left((\Delta e)^{\frac{1}{r+2}}+(\Delta e)^{\frac{1}{p+2}}\right) .
\end{aligned}
$$

Hence, we have from (4.55) and the definition of $A_{4}(t)^{2}$

$$
e(t) \leq C\left\{\Delta e+(\Delta e)^{\frac{1}{r+2}}+(\Delta e)^{\frac{2(r+1)}{r+2}}+(\Delta e)^{\frac{1}{p+2}}\right\} \leq C(\Delta e)^{\kappa_{4}},
$$

where $\kappa_{4}=\min \left\{\frac{1}{r+2}, \frac{2(r+1)}{r+2}, \frac{1}{p+2}\right\}$. Therefore,

$$
\sup _{t \leq s \leq t+T} e(s)^{\frac{1}{\kappa_{4}}} \leq C(e(t)-e(t+T)) .
$$

Applying Lemma 2.2 to (4.62) we conclude

$$
e(t) \leq C(1+t)^{-\eta_{4}}
$$

with $\eta_{4}=\min \left\{\frac{1}{r+1}, \frac{-r}{2(r+1)}, \frac{1}{p+1}\right\}$.

Now, the proof of the Theorem 2.2 is complete.

\section{References}

[1] S. Berrimi, S. A. Messaoudi, Exponential decay of solutions to a viscoelastic equation with nonlinear localized damping, Electron. J. Differential Equations, 2004 (88) (2004) 1-10.

[2] M. M. Cavalcanti, Existence and uniform decay for Euler-Bernoulli viscoelastic equation with nonlocal boundary dissipation, Discrete Contin. Dyn. Syst., 8 (3) (2002) 675-695.

[3] M. M. Cavalcanti, V. N. Cavalcanti, T. F. Ma, Exponential decay of the viscoelastic EulerBernoulli equation with a nonlocal dissipation in general domains, Differential Integral Equations, 17 (5-6) (2004) 495-510.

[4] R. C. Charão, E. Bisognin, V. Bisignin, A. F. Pazoto, Asymptotic behavior of a BernoulliEuler type equation with nonlinear localized damping, Progr. Nonlinear Differential Equations Appl., 66 (2006) 67-91.

[5] C. M. Dafermos, Energy methods for non linear hyperbolic Volterra integro-differential equation, Comm. P. D. E., 3 (1979) 119-178.

[6] J. U. Kim, A unique continuation property of a beam equation with variable coefficients, Estimation and control of distributed parameter systems, (1991) 197-205.

[7] J. U. Kim, Exact controllability of an Euler-Bernoulli equation, SIAM J. Control Optm., 30 (1992) 1001-1023.

[8] J. Lagnese, Boundary stabilization of thin plates in "SIAM Studies in Applied Mathematics", SIAM, Philadelphia, PA, 1988.

[9] I. Lasiecka, Exponential decay rates for the solutions of Euler-Bernoulli equation with boundary dissipation occurring in the moments only, J. Differential equations, 95 (1992) 169-182. 
[10] I. Lasiecka, R. Triggiani, Sharp Trace Estimates of solutions to Kirchoff and Euler-Bernoulli equations, Appl. Math. Optim., 28 (1993) 277-306.

[11] J. L. Lions, Exact controllability, stabilization and perturbations for distributed systems, SIAM Rev., 30 (1988) 1-68.

[12] J. L. Lions, E. Magenes, Problèmes aux limites non homogènes Vol. 1. Aplications, Dunod, Paris, 1968.

[13] M. Nakao, A difference inequality and application to nonlinear evolution equations, J. Math. Soc. Japan, 30 (1978) 747-762.

[14] M. Nakao, Decay of solutions of the wave equation with a local nonlinear dissipation, Math. Ann., 305 (1996) 403-417.

[15] M. Nakao, Il Hyo Jung, Energy decay for the wave equation in exterior domain with some half-linear dissipation, Differential Integral Equations, 16 (8) (2003) 927-948.

[16] M. Nakao, Jeong Ja Bae, Energy decay and periodic solution for the wave equation in an exterior domain with half-linear and nonlinear boundary dissipations, Nonlinear Anal., 66 (2007) 301-323.

[17] J. C. Oliveira, R. C. Charão, Stabilization of a locally damped incompressible wave equation, J. Math. Anal. Appl., 303 (2005) 699-725.

[18] J. Y. Park, T. G. Ha, Energy decay for nondissipative distributed system with boudary damping and source term, Nonlinear Anal., 70 (2009) 2416-2434.

[19] J. Y. Park, T. G. Ha, Existence and asymptotic stability for the semilinear wave equation with boundary damping and source term, J. Math. Phys., 49 (2008) 053511.

[20] J. Y. Park, J. A. Kim, Existence and uniform decay for Euler-Bernoulli beam equation with memory term, Math. Meth. Appl. Sci., 27 (2004) 1629-1640.

[21] M. Tucsnak, Semi-internal stabilization for a non-linear Bernoulli-Euler equation, Math. Meth. Appl. Sci., 19 (1996) 897-907.

[22] E. Zuazua, Exponential decay for the semilinear wave equation with locally distributed damping, Comm. P.D.E., 15 (1990) 205-235.

Department of Mathematics, Pusan National University, Busan 609-735, KOREA

E-mail address: htg@pusan.ac.kr

Department of Mathematics, Pusan National University, Busan 609-735, KOREA

E-mail address: jyepark@pusan.ac.kr 